\title{
A Nonlinear Updated Gain Observer for MIMO Systems: Design, Analysis and Application to Marine Surface Vessels
}

\begin{abstract}
In this paper, the state estimation problem of a class of multi-input-multi-output nonlinear systems with measurement noise is studied. We develop an extended updated-gain high gain observer to make a tradeoff between reconstruction speed and measurement noise attenuation. The designed observer, whose gains are driven by nonlinear functions of the available output estimation errors, has the ability to reconstruct system states quickly and reduce the effect of measurement noise. We establish that, if there exists a state feedback law exponentially stabilizing the system with respect to an invariant set, the estimations and estimation errors are bounded. Besides, the trajectories of state- and output-feedback (based on the proposed observer) are sufficiently close, namely performance recovery. The observer performance is illustrated by various examples in marine control, including a case of transformation into the predefined structure.
\end{abstract}

Keywords: high-gain observers, nonlinear systems, measurement noise, marine control.

\section{Introduction}

The observer design is undoubtedly crucial in mechanical control systems [1]. High-gain observer, which has the ability to recover performance quickly, is a general approach to construct state observers for nonlinear systems [2,3]. It is well-known that measurement noise 5 in mechanical systems leads to a tradeoff between the state reconstruction speed and noise attenuation $[4,5]$. In the transient stage, observers should provide fast state reconstructions; when estimation errors are relatively small, observers are expected to have the ability of noise attenuation. To acquire the demanded performance, many scholars hold the view that an available approach is updating gains of observers dynamically. Recently, some remarkable efforts have been devoted to design high-gain observers with updated or adaptive gains [5-9]. Ahrens and Khalil propose a switched-gain approach between two gain values for a class of nonlinear systems in the feedback linearization framework with a chain of integrators [5]. In their work, 
a delay time is incorporated to prevent frequent gain switchings. Sanfelice and Praly present a high-gain observer with gain adaption for a class of uniformly observable nonlinear systems with a strict-feedback structure, and they provide the bound on the mean of error signals, utilized to analyze performance and effects of measurement noise [6]. Compared with setting a delay time in [5], the adaptive law of observer gains is more feasible to implement practically. One major drawback of this approach is that estimation errors exhibit highly oscillations in [6]. On that basis, a nonlinear-gain structure is proposed to make a tradeoff between the speed of state reconstruction and noise attenuation in [7, whose system model is in the same form as [5]. The nonlinear-gain structure has the ability to overcome the highly oscillatory behavior with a relatively large root of the characteristic equation, yielding a two-time-scale structure which guarantees that the output estimation comes into an invariant set monotonously. SISO nonlinear systems are considered in the aforementioned observers.

These forms of nonlinear observers are not applicable for numerous mechanical systems for several reasons: (1) some mechanical systems are depicted by MIMO structures with environmental disturbances and measurement noise; (2) a variety of mechanical systems do not have the above-motioned structures due to complicated coordinate transformations. Hence, studies on nonlinear observers with measurement noise are not limited in SISO systems, and some valuable efforts have been devoted to nonlinear MIMO systems. An updated-gain adaptive law is introduced for two kinds of MIMO systems which are characterized by a triangular canonical form and a non-triangular form, in [8, 9] respectively, with a proper scalar Riccati differential equation. One of the limitations with this approach is that the model is in absence of unknown exogenous input. Nevertheless, uncertainties and disturbance estimations are particularly crucial for system control and disturbance attenuation [10, 11].

However, few precise results exist for high-gain observer of nonlinear MIMO systems with measurement noise and environmental disturbances. This paper attempts to fill the gap. Motivated by [6] and [7], an extended updated-gain high gain observer is proposed for a class of MIMO systems with measurement noise in this paper. The form of MIMO systems is given in Section 2, which can be regarded as the MIMO version of the structure in [7]. Unlike in the above-mentioned contributions [8, 9] where Riccati differential equations are utilized, the gains in the paper are driven by nonlinear functions of the output observation errors. Due to the low accuracy of identified model parameters in mechanical systems, an extended vector 
is utilized to estimate modeling errors instead of the boundness assumption of the estimation term $\bar{\delta}$ in [7], also removing the prescient requirement of accurate model information in [9]. The proposed observer has the ability to reconstruct states quickly in the transient stage and suppress measurement noise in the steady-state stage. To validate the effectiveness of the proposed observer, two marine surface vessel models in [12 and [13] are analyzed. In fact, the second model does not have the predefined structure in the feedback linearization frame. With a transformation approach, the proposed observer can be utilized for the underactuated path following model.

The remainder of the paper is organized as follows. Section 2 is devoted to the form of a class of MIMO systems and some technical backgrounds. The observer design and main results are shown in Section 3, In Section 4, various examples in marine control illustrate the method. Simulation results are also presented in Section 4. To improve readability and legibility, all proofs of lemmas and theorem are given in Appendices. Symbols and notations are given in Table 1

Table I: Notations

\begin{tabular}{ll|ll}
\hline$\|\cdot\|$ & the norm of a vector & $|\cdot|$ & the norm of a scalar \\
\hline$\|\cdot\|_{\mathcal{I}}$ & the common point-to-set distance & $\|\chi\|_{\mathcal{I}}=d(\chi, \mathcal{I})=\inf _{x_{0} \in \mathcal{I}} d\left(\chi, x_{0}\right)$ \\
\hline$\lambda_{\min }(A)$ & the minimal eigenvalue of matrix $A$ & $\lambda_{\max }(A)$ & the maximal eigenvalue of matrix $A$ \\
\hline $\mathcal{O}(\cdot)$ & order of magnitude notation & $\cdots \cdot "$ & the time derivative of a function \\
\hline $\mathcal{L}_{f} h$ & $\begin{array}{l}\text { the Lie derivative of } h \text { with respect } \\
\text { to the vector filed } f\end{array}$ & $\mathcal{C}^{1}$ & $\begin{array}{l}\text { the class of continuously differentiable } \\
\text { functions }\end{array}$ \\
\hline $\mathcal{C}^{0}$ & the class of continuous functions & & \\
\hline
\end{tabular}

\section{Problem Formulation}

Consider a class of MIMO nonlinear systems in the feedback linearization framework, which are diffeomorphic to the following form:

$$
\begin{aligned}
\dot{z} & =f_{0}(x, z)+g_{1}(t, \zeta) \\
\dot{x} & =A x+B\left[\psi(x, z, u)+g_{2}(t, \zeta)\right] \\
y & =C x+\ell \\
\omega & =\Theta(x, z, \zeta)
\end{aligned}
$$


where $x \in \mathbb{R}^{n}$ and $z \in \mathbb{R}^{m}$ are states, $u \in \mathbb{R}^{p}$ is the control input, $y \in \mathbb{R}^{p}$ and $\omega \in \mathbb{R}^{s}$ are measured output vectors, $\zeta \in \mathbb{R}^{q}$ denotes the unknown exogenous input, and $\ell \in \mathbb{R}^{p}$ denotes measurement noise. Matrices are given by

$$
\begin{aligned}
& A=\operatorname{blockdiag}\left(A_{1}, \ldots, A_{p}\right), B=\operatorname{blockdiag}\left(B_{1}, \ldots, B_{p}\right), C=\operatorname{blockdiag}\left(C_{1}, \ldots, C_{p}\right), \\
& A_{i}=\left[\begin{array}{ccccc}
0 & 1 & 0 & \cdots & 0 \\
0 & 0 & 1 & \cdots & 0 \\
\vdots & \vdots & \ddots & \ddots & \vdots \\
0 & 0 & \cdots & 0 & 1 \\
0 & 0 & \cdots & 0 & 0
\end{array}\right]_{n_{i} \times n_{i}}, B_{i}=\left[\begin{array}{c}
0 \\
\vdots \\
0 \\
1
\end{array}\right]_{n_{i} \times 1}, \text { and } C_{i}=\left[\begin{array}{llll}
1 & 0 & \cdots & 0
\end{array}\right]_{1 \times n_{i}},
\end{aligned}
$$

where $i=1, \ldots, p, n=\sum_{i=1}^{p} n_{i}$, and $(A, B, C)$ represents $p$ chains of integrators.

The MIMO feedback linearization model has a large number of applications in mechanical control systems, including guidance laws of missiles, flight control models, robot manipulators, roll stabilization of ships, etc [14 21]. $g_{1}(t, \zeta)$ and $g_{2}(t, \zeta)$ are disturbance terms, which are commonly regarded as affine terms in mechanical systems [13, 22]. Before proposing our main results, some assumptions need to be made as follows, which are justifiable for most mechanical systems.

\section{Assumption 1.}

- $\zeta(t) \in \mathcal{D}$ is a differential exogenous input, where $\mathcal{D}$ is a compact set in $\mathbb{R}^{q}$;

- Perturbation terms $g_{1}(\cdot, \cdot), g_{2}(\cdot, \cdot) \in \mathcal{C}^{1}$ are locally Lipschitz in their arguments over $\left[t_{0},+\infty\right) \times \mathcal{D}$, and $g_{1}(t, 0)=0, g_{2}(t, 0)=0 ;$

- $\ell(t)$ is bounded and continuous, and $\sup _{t \in\left[t_{0},+\infty\right)}\|\ell(t)\|=\bar{\ell}$;

- The mappings $f_{0}, \psi$ and $\Theta$ are locally Lipschitz and continuous in their arguments over the domain of interest; besides, the mapping $\psi(x, z, \zeta) \in \mathcal{C}^{1}$.

There is a continuous state feedback control law as follows:

$$
\begin{aligned}
& \dot{\theta}=\Phi(\theta, x, \omega, \zeta) \\
& u=\gamma(\theta, x, \omega, \zeta)
\end{aligned}
$$

where $\theta \in \mathbb{R}^{n_{\theta}}$ and $u \in \mathbb{R}^{p}$. The closed-loop system with the above state feedback law is

$$
\dot{\chi}=f_{s}(\chi, \zeta)
$$


where $\chi=\left[\begin{array}{lll}x & z & \theta\end{array}\right]^{\top}$ and

$$
f_{s}(\chi, \zeta)=\left[\begin{array}{c}
A x+B\left[\psi(x, z, \gamma(\theta, x, \omega, \zeta))+g_{2}(t, \zeta)\right] \\
f_{0}(x, z)+g_{1}(t, \zeta) \\
\Phi(\theta, x, \omega, \zeta)
\end{array}\right]
$$

\section{Assumption 2.}

- The autonomous system $\dot{\chi}=f_{s}(\chi, \zeta)$ is globally uniformly asymptotically stable with respect to an invariant set $\mathcal{I}$;

- The vector field in (2), $\Phi$ and $\gamma$, are locally Lipschitz in their arguments, uniformly in $\zeta$, over their domain of interest.

Remark 1. It is worth noticing that the control input $u \in \mathcal{C}^{0}$ due to Assumption 2. Asymptotical stability with respect to $\mathcal{I}$ is common in mechanical systems with bounded exogenous disturbances. A stable system is of possibility to be asymptotically stable with respect to $\mathcal{I}$ in the presence of perturbations under reasonable assumptions. It is summarized as Lemma 1 .

Lemma 1. Suppose the origin is the globally exponentially stable equilibrium of $\dot{\chi}=f_{s}(\chi, 0)$. If $\left.\sup _{(t, \zeta) \in\left[t_{0},+\infty\right) \times \mathcal{D}} \max \left\{\left\|g_{1}(t, \zeta)\right\|,\left\|g_{2}(t, \zeta)\right\|\right)\right\}=\bar{g}_{0}<+\infty$, the perturbed system $\dot{\chi}=f_{s}(\chi, \zeta)$ is globally uniformly asymptotically stable with respect to an invariant set $\mathcal{I}$, where

$$
\mathcal{I}=\left\{\chi \mid\|\chi\|<\bar{g}_{0} c_{4} /\left(\theta c_{3}\right)\right\}
$$

Proof. See Appendix A.

The following lemma is instrumental in the proof of our main results. A similar version of Lemma 2 is given in [5]. The differences between two lemmas, caused by the extended states in the proposed observer, should be noticed.

Lemma 2. Give a two-dimensional function $F_{r}\left(\varepsilon_{2}, \bar{\ell}\right)=\kappa_{1} \varepsilon_{2}^{-n^{*}+1} \bar{\ell}+\kappa_{2} \varepsilon_{2}$, where $\kappa_{1}, \kappa_{2}>0$, $n^{*}$ is a positive integer and $n^{*} \geq 2$. For $\varepsilon_{2} \in(0,+\infty)$ and $\bar{\ell} \in[0,+\infty)$, the function $F_{r}\left(\varepsilon_{2}, \bar{\ell}\right)$ has the following properties. (1) For a given $\bar{\ell}$, the function $F_{r}\left(\varepsilon_{2}, \bar{\ell}\right)$ gets its minimal value at $\varepsilon_{2}=\left(n^{*} \kappa_{1} \bar{\ell} / \kappa_{2}\right)^{1 /\left(n^{*}+1\right)} \triangleq \kappa_{a} \bar{\ell}^{1 /\left(n^{*}+1\right)}$, and $\min _{\varepsilon_{2}>0} F_{r}\left(\varepsilon_{2}, \mu\right)=\left(\kappa_{1} / \kappa_{a}^{n^{*}}+\kappa_{2} \kappa_{a}\right) \bar{\ell}^{1 /\left(n^{*}+1\right)}$. For a given $\bar{\ell}, F_{r}\left(\varepsilon_{2}, \bar{\ell}\right)$ is a strictly increasing function of $\varepsilon_{2}$ if $\varepsilon_{2} \in\left(\kappa \bar{\ell}^{1 /\left(n^{*}+1\right)},+\infty\right)$, and 
100

$F_{r}\left(\varepsilon_{2}, \bar{\ell}\right) \leq \kappa_{b} \varepsilon_{2}$ where $\kappa_{b}=\kappa_{1}+\kappa_{2} / \kappa_{a}^{\left(n^{*}-1\right)}$. (3) For a given $k^{*}>0, \forall \bar{\ell} \in\left[0,\left(k^{*} / \kappa_{a}\right)^{n^{*}+1}\right)$, there exist $\varepsilon_{m}\left(\bar{\ell}, k^{*}\right)$ and $\varepsilon_{M}\left(\bar{\ell}, k^{*}\right)$ satisfying $\varepsilon_{m} \in\left[0, \kappa \bar{\ell}^{1 /\left(n^{*}+1\right)}\right)$ and $\varepsilon_{M}>\kappa_{a} \bar{\ell}^{1 /\left(n^{*}+1\right)}$ such that $F_{r}\left(\varepsilon_{2}, \bar{\ell}\right) \leq k^{*}, \forall \varepsilon_{2} \in\left(\varepsilon_{m}, \varepsilon_{M}\right)$.

Proof. It is omitted as similar to the proof of Lemma 1 in [5].

\section{Observer Design and Main Results}

In this section, we present our main result of the updated-gain high-gain observer for a class of MIMO systems as (1), to handle the tradeoff between state reconstruction speed and measurement noise attenuation. To estimate state $x(t)$, a full-order observer is designed as

$$
\dot{\hat{x}}=A \hat{x}+B[\hat{\psi}(\hat{x}, \hat{z})+\hat{\Sigma}]+H\left(\varepsilon_{1}, \varepsilon_{2}, y-C \hat{x}\right)
$$

where $\hat{x}(t)$ is the estimation of $x(t)$, and

$$
\begin{gathered}
\hat{\Sigma}=\left[\begin{array}{c}
\hat{\sigma}_{1} \\
\vdots \\
\hat{\sigma}_{p}
\end{array}\right], \dot{\hat{\sigma}}_{i}=\left(\frac{\alpha_{i, n_{i}+1}}{\varepsilon_{\left(i\left(n_{i}+1\right)\right)}(t)^{n_{i}+1}}\right) \cdot(y-C \hat{x})_{i} \\
H\left(\varepsilon_{1}, \varepsilon_{2}\right)=\left[H_{1}^{\top}\left(\varepsilon_{1}, \varepsilon_{2}\right), \cdots, H_{p}^{\top}\left(\varepsilon_{1}, \varepsilon_{2}\right)\right]^{\top}, H_{i}\left(\varepsilon_{1}, \varepsilon_{2}\right)=\left[\begin{array}{c}
h_{i 1}(y-C \hat{x})_{i} \\
\vdots \\
h_{i n_{i}}(y-C \hat{x})_{i}
\end{array}\right], i=1, \ldots, p \\
h_{i j}(y-C \hat{x})_{i}=\frac{\alpha_{i j}}{\varepsilon_{(i j)}(t)^{j}}(y-C \hat{x})_{i}, j=1, \ldots, n_{i}
\end{gathered}
$$

where for $j=1, \ldots, n_{i}+1$,

$$
\varepsilon_{(i j)}(t)= \begin{cases}{\left[\frac{1}{\varepsilon_{1}^{j}}+d_{i}\left(\frac{1}{\varepsilon_{2}^{j}}-\frac{1}{\varepsilon_{1}^{j}}\right) \cdot \frac{\operatorname{sat}\left(\frac{(y-C \hat{x})_{i}}{d_{i}}\right)}{(y-C \hat{x})_{i}}\right]^{-\frac{1}{j}},} & (y-C \hat{x})_{i} \neq 0 \\ \varepsilon_{2}, & (y-C \hat{x})_{i}=0\end{cases}
$$

110

\section{$\psi$} a nonlinear function with updated gains driven by the output estimated errors. $\hat{\Sigma}(\cdot)$ is an extended state vector to estimate the environmental perturbations caused by disturbances, unmodeled dynamics and model uncertainties. 
Remark 2. We use $(\cdot)_{i}$ to denote the $i$-th element of $(\cdot)$, and denote $x_{i j} \triangleq x_{\left(j+\sum_{k=0}^{i-1} n_{k}\right)}, \hat{x}_{i j} \triangleq$ $\hat{x}_{\left(j+\sum_{k=0}^{i-1} n_{k}\right)}$ for simplicity. If $(y-C \hat{x})_{i} \in\left[0, d_{i}\right], \varepsilon(t)=\varepsilon_{2} ; \lim _{\left\|(y-C \hat{x})_{i}\right\| \rightarrow \infty} \varepsilon(t)=\varepsilon_{1}$. Suppose $0<\varepsilon_{1} \ll \varepsilon_{2}<1$, thus $\varepsilon_{(i j)}(t) \in\left(\varepsilon_{1}, \varepsilon_{2}\right]$. If $(y-C \hat{x})_{i}$ is outside the set $\left[-d_{i}, d_{i}\right]$, the corresponding $1 / \varepsilon_{i *}(t)$ is relatively large, yielding a fast transient reconstruction; when $(y-$ $C \hat{x})_{i}$ keeps in the set $\left[-d_{i}, d_{i}\right]$, the observer has lower gains, reducing the effect of measurement noise on estimations. It should be satisfied that the set $\Omega_{2}=\left\{\left|y_{1}-\hat{x}_{11}\right|<d_{1}\right\} \times \ldots \times\left\{\mid y_{p}-\right.$ $\left.\hat{x}_{p 1} \mid<d_{p}\right\}$ is an invariant set, and the trajectory of $y-C \hat{x}$ comes into to $\Omega_{2}$ monotonically.

\section{Assumption 3.}

- The roots of $s^{n_{i}+1}+\alpha_{i, 1} s^{n_{i}}+\ldots+\alpha_{i, n_{i}} s+\alpha_{i, n_{i}+1}=0(i=1, \ldots, p)$ are real numbers in the left half plane (LHP), and each equation has one fast root whose absolute value is relatively large.

- $\varepsilon_{1}$ and $\varepsilon_{2}$ are positive constants, and $0<\varepsilon_{1} \ll \varepsilon_{2}<1$. Denote $\varepsilon_{f} \triangleq \varepsilon_{1} / \varepsilon_{2}$, and $\varepsilon_{f} \in(0,1 / 2)$.

- The term $\hat{\psi}(\cdot, \cdot)$ is locally Lipschitz in its argument and $\hat{\psi} \in \mathcal{C}^{1}$.

In order to guarantee the estimation trajectory comes into $\Omega_{2}$ monotonically, the absolute value ration of the fast root and slow roots should be more than 10 for each equation. The uniform constraint of $\|\psi-\hat{\psi}\|$ is removed by the extended term $\hat{\Sigma}$, allowing relatively significant identification errors of system parameters. Parameters $\varepsilon_{1}$ and $\varepsilon_{2}$ are suggested to be selected according to the performance of conventional linear-gain high gain observers, where $\varepsilon_{1}$ is related to the transient response and the efficiency of measurement noise attenuation is depended on $\varepsilon_{2}$. Besides, the values of $\left(d_{i}\right)$ s should be chosen small enough with the lower bounds $d_{i}>\ell_{i}$. The proposed observer yields the following theorem.

Theorem 1. Consider the MIMO system (1) with the continuous state feedback control law (2), and let Assumptions 1, 3 hold. Suppose $\chi\left(t_{0}\right) \in U_{0}$ and $\hat{x}\left(t_{0}\right) \in U_{0}^{*}$, where $U_{0}$ and $U_{0}^{*}$ are two compact sets with feasible dimensions. There is a positive constant $\lambda^{*}$ such that for all the fast roots in $\left(-\infty, \lambda^{*}\right)$, the closed-loop system with the output feedback law based on the observer (6) has the following properties: (1) there is no escape time in the estimation error vector $e(t) \triangleq x(t)-\hat{x}(t)$, and the Carathèodory solution $t \rightarrow(\hat{x}, e)$ is bounded; (2) the ultimate bound of extended estimation errors, $\max \{\|x(t)-\hat{x}(t)\|,\|\Delta(t)\|\}$, is no more 
than $F_{r}\left(\varepsilon_{2}, \bar{\ell}\right)$ after the moment $t_{0}+T\left(\varepsilon_{1}, \varepsilon_{2}\right)$, and $T\left(\varepsilon_{1}, \varepsilon_{2}\right) \rightarrow 0$ as $\varepsilon_{2} \rightarrow 0$. For a given $\bar{\ell}$, the ultimate bound $F_{r}\left(\varepsilon_{2}, \bar{\ell}\right)$ gets the minimal value at $\kappa_{a} \bar{\ell}^{1 /\left(n^{*}+1\right)} ;(3)$ if the measurement noise vector $\bar{\ell} \in\left[0, \min \left(\ell_{1}^{*}, \ell_{2}^{*}, \ell_{3}^{*}\right)\right)$ and $\varepsilon_{2} \in\left(\kappa \bar{\ell}^{1 /\left(n^{*}-1\right)}, \min \left(\varepsilon_{M}, \varepsilon_{2}^{(1)}\right)\right)$, the states of the output feedback system based on the proposed observer are bounded, satisfying $\|\chi(t)\|_{\mathcal{I}} \leq \chi^{*} ;$ (4) there exist $\chi_{e}^{*}>0$ and a finite time $\mathcal{T}$ such that $\left\|\chi(t)-\chi_{r}(t)\right\| \leq \chi_{e}^{*}\left(\varepsilon_{1}, \varepsilon_{2}\right)$ for $t \in\left[t_{0}+\mathcal{T},+\infty\right)$ with the same initial values of $\chi$ and $\chi_{r}$.

Proof. See Appendix B. We give an outline of the proof here. Firstly, we will demonstrate that there is no escape time in the extended estimation error vector $e(t)$. It will be shown that a class $\mathcal{K}$ function of $e(t)$ cannot grow faster than exponentially, yielding no escape time in $e(t)$. Secondly, a scaled vector of $e(t)$ is proved to be exponentially convergent and ultimately bounded, and the ultimate bound is given w.r.t. $\varepsilon_{1}, \varepsilon_{2}$ and $\bar{\ell}$. In fact, it reveals that the trajectory of $y-C \hat{x}$ comes into $\Omega_{2}$ monotonically. Thirdly, the system states are proved to keep bounded with output feedback laws based on the observer. Finally, we demonstrate the ability of performance recovery, that is $\chi(t)$ and $\chi_{r}(t)$ are close enough.

Remark 3. The full system structure with output feedback law based on the proposed observer is illustrated in Fig. 1. The first property of Theorem 1 is related to the well-known peaking phenomenon, which exhibits an impulsive-like behavior. Such property means that the estimation error will not be driven infinitely. The second one gives the ultimate bound of $e(t)$ after a short time $T\left(\varepsilon_{1}, \varepsilon_{2}\right)$, and the time interval $T\left(\varepsilon_{1}, \varepsilon_{2}\right)$ could be arbitrarily small. It shows that the parameter $\varepsilon_{2}$ should be selected surrounding $\kappa_{a} \bar{\ell}^{1 /\left(n^{*}+1\right)}$ to guarantee the steady-state errors small enough. $\|\chi(t)\|_{\mathcal{I}} \leq \chi^{*}$ emphasizes that the observer keeps the boundness of the original autonomous system with state feedback. The fourth term of Theorem 1 ensures performance recovery of the proposed observer, which means the trajectories with state- and output feedback are close enough for $t \in\left[t_{0}+\mathcal{T},+\infty\right)$.

Remark 4. $\varepsilon_{2} \in(0,1)$ determines the steady-state stage estimation errors, and relatively small errors can be realized by selecting $\varepsilon_{2}$ around $\kappa \bar{\ell}^{1 /\left(n^{*}+1\right)}$. The state reconstruction speeds are related to $\varepsilon_{1}$. A sufficiently large $\varepsilon_{1}$ causes significant peaking phenomena, which should 170 be solved by input or state saturations. For a given $i$, the selection of $\alpha_{i j}$ s is equivalent to choosing the roots of equation $s^{n_{i}+1}+\alpha_{i, 1} s_{i}^{n}+\ldots+\alpha_{i, n_{i}+1}=0$, which are all in the LHP. If the absolute value of the fastest root is not large enough, the output error $y-C \hat{x}$ cannot come into the predesigned invariant set monotonically, causing the oscillatory behavior of 
updated gains. Furthermore, $d_{i}$ s resolve the moment of gain updating. If $d_{i}$ s are too large,

the proposed observer degenerates into the conventional linear high-gain observer for MIMO nonlinear systems with $\varepsilon=\varepsilon_{2}$. Conversely, sufficiently small $d_{i}$ s turn the observer into a linear high-gain observer with $\varepsilon_{1}$, yielding large estimation errors in the steady-state stage.

\section{Examples in Marine Control}

This section gives two design examples in marine control, rudder roll stabilization and

$$
\begin{aligned}
& \left(m+m_{x}\right) \dot{u}-\left(m+m_{y}\right) v r=X \\
& \left(m+m_{y}\right) \dot{v}+\left(m+m_{x}\right) u r+m_{y} \alpha_{y} \dot{r}-m_{y} l_{y} \dot{p}=Y \\
& \left(I_{x}+J_{x}\right) \dot{p}-m_{y} l_{y} \dot{v}-m_{x} l_{x} u r+W G M \phi=K \\
& \left(I_{z}+J_{z}\right) \dot{r}+m_{y} \alpha_{y} \dot{v}=N-Y x_{G}
\end{aligned}
$$

where $m, I_{x}$ and $I_{z}$ are the mass and moment of inertia of a vessel. $m_{x}, m_{y}, J_{z}$ and $J_{x}$ are the added mass and added moment of inertia. $W$ is the vessel displacement, and $\overline{G M}$ is the metacentric height. $l_{x}$ and $l_{y}$ are the $z$-coordinates of the centers of $m_{x}$ and $m_{y}$, respectively; $\alpha_{y}$ denotes the $x$-coordinate of the center of $m_{y} ; x_{G}$ is the $x$ coordinate of the gravity center. $[u, v, \omega]^{\top}$ and $[p, q, r]^{\top}$ denote the translational velocities and angular velocities (surge, sway, heave, roll, pitch and yaw), respectively. $X$ and $Y$ denote hydrodynamic forces in the $x$ direction and the $y$-direction; $K$ and $N$ denote hydrodynamic moments about the $x$-axis and the $z$-axis. Besides, these hydrodynamics are affected by environmental disturbances (wind, waves and ocean currents) and the rudder angle $\delta$ which is the control input.

The purpose of roll stabilization is to design a rudder control law $\delta(\cdot)$ stabilizing the states $\psi, \phi, p$ and $r$ w.r.t an invariant set. The surge velocity $u$ is supposed to be a constant. Notice that $\dot{\phi}=p$ and $\dot{\psi}=r$, yielding a structure with two chains of integrators. $v$ and environmental 
disturbances can be regarded as the unknown exogenous input vector $\zeta(t)$ in Section 2 Angles $\psi$ and $\phi$ are detectable with measurement noise as

$$
y_{1}=\psi+\ell_{1}, y_{2}=\phi+\ell_{2}
$$

195 ed. 23] gives a state feedback law for rudder roll stabilization (RRS), where states $\phi, \psi, p$ and $r$ exponentially converge into an invariant set containing the origin in the presence of environmental disturbances. Thus according to Theorem 1 , an observer is designed to estimate $r$ and $q$ as follows.

$$
\begin{aligned}
\dot{\hat{\psi}} & =\hat{r}+\frac{\alpha_{11}}{\varepsilon_{(11)}(t)}\left(y_{1}-\hat{\psi}\right) \\
\dot{\hat{\phi}} & =\hat{p}+\frac{\alpha_{21}}{\varepsilon_{(21)}(t)}\left(y_{2}-\hat{\phi}\right) \\
\dot{\hat{r}} & =f_{2}(0, \hat{r}, \hat{\phi}, \hat{p}, \delta)+\hat{x}_{5}+\frac{\alpha_{12}}{\varepsilon_{(12)}^{2}(t)}\left(y_{1}-\hat{\psi}\right) \\
\dot{\hat{p}} & =\frac{1}{\tilde{a}_{34}} \cdot f_{3}(0, \hat{r}, \hat{\psi}, \hat{p}, \delta)+\hat{x}_{6}+\frac{\alpha_{22}}{\varepsilon_{(22)}^{2}(t)}\left(y_{2}-\hat{\phi}\right) \\
\dot{\hat{x}}_{5} & =\frac{\alpha_{13}}{\varepsilon_{(13)}^{3}(t)}\left(y_{1}-\hat{\psi}\right) \\
\dot{\hat{x}}_{6} & =\frac{\alpha_{23}}{\varepsilon_{(23)}^{3}(t)}\left(y_{2}-\hat{\phi}\right)
\end{aligned}
$$
$0, r(0)=0, p(0)=0, \hat{\psi}(0)=0, \bar{\phi}(0)=0, \hat{r}(0)$ and $\hat{p}(0)=0$. The angular velocities $p$ and $r$ 
are undetectable, the estimated states of which are given in Fig. 3. The simulation results of Example 1 show that the proposed observer can reconstruct the undetectable states quickly, verifying the first two terms in Theorem 1. More about parameter selections and performance recovery will be discussed in Example 2.

\subsection{Example 2: Path following (Li, Sun \&5 Oh, 2009) [13]}

The second example is path following of underactuated marine surface vessels, the model of which is borrowed from [13], in regard to demonstrate the ability of performance recovery.

Considering environmental disturbances and unmodeled dynamics with position and orientation measurements only, the dynamics are given as (13). The model is widely used in research [13, 24, 26].

$$
\begin{aligned}
& \dot{\bar{\psi}}=r, \dot{e}=u \sin \bar{\psi}+v \cos \bar{\psi} \\
& \dot{v}=-\frac{m_{11}}{m_{22}} u r-\frac{d_{22}}{m_{22}} v+\tau_{1}, \dot{r}=\frac{m_{11}-m_{22}}{m_{33}} u v-\frac{d_{33}}{m_{33}} r+\frac{1}{m_{33}} u_{2}+\tau_{2} \\
& y_{1}=\bar{\psi}+\ell_{1}, y_{2}=e+\ell_{2}
\end{aligned}
$$

where $\bar{\psi}$ is the cross-track error. $u_{2}$ is the yaw moment which is the control input. The parameters $m_{i i}$ and $d_{i i}$ are given by vessel inertia, added mass effects and hydrodynamic dampings. $\tau_{1}(t)$ and $\tau_{2}(t)$ are time-varying environmental disturbances. $\ell_{1}$ and $\ell_{2}$ are measurement noise of $\bar{\psi}$ and $e$, satisfying Assumption 1 , thus $\dot{y}_{2}^{-}$well-defined. $y=\left[y_{1}, y_{2}\right]^{\top}$ is the measured output, and suppose that $y(t)$ is derivable a.e.. The surge velocity $u$ is usually assumed to be a constant [13, 23, 27]. More details about this model could be found in [13].

In the model, guidance dynamics, motion dynamics are in two-time scales. States $\bar{\psi}$ and $e$ are in the slow subsystem, and $v$ and $r$ are in the fast one. It is obvious that the model (13) is not in the predefined form as (1). In order to get a structure with two chains of integrators, we define a virtual variable $x_{v}$ satisfying $\dot{x}_{v}=v$. However, the virtual variable $x_{v}$ is undetectable. Thus a technique is given to estimate the value of $x_{v}$ with the multi-time-scale structure. According to the geometric relationship, we have $x_{v}(t)=\int_{t_{0}}^{t}\left(\frac{\dot{e}}{\cos \psi}-u \tan \bar{\psi}\right) \mathrm{d} \tau$ with $x_{v}\left(t_{0}\right)=x_{v 0}$. The initial value $x_{v 0}$ has no effect on the estimation of $v$. It is easy to verify that $x_{v}$ will come into an invariant set if the autonomous system with state feedback is exponentially stable w.r.t an invariant set $\mathcal{I}$. The "measured output" $x_{v}$, which is defined as $y_{3}$, is computed by $y_{1}$ and $y_{2}$, and $y_{3}(t)=\int_{t_{0}}^{t} \dot{y}_{2}^{-} / \cos y_{1}-u \tan y_{1} \mathrm{~d} \tau$, illustrated by Fig. 2 . 
According to the assumptions of $\ell_{1}$ and $\ell_{2}$, for $t \in\left[t_{0}, t_{0}+t^{*}\right]$,

$$
\begin{aligned}
y_{3}-\left.x_{r}\right|_{t} & =\int_{t_{0}}^{t}\left(\frac{\overbrace{e+l_{2}}-u \sin \left(\bar{\psi}+\ell_{1}\right)}{\cos \left(\bar{\psi}+\ell_{1}\right)}-\frac{\dot{e}-u \sin \bar{\psi}}{\cos \bar{\psi}}\right) \mathrm{d} \tau \\
& \leq\left\|e\left(t_{0}+t^{*}\right)-e\left(t_{0}\right)\right\| \int_{t_{0}}^{t_{0}+t^{*}}(1-\cos \bar{\ell}+\sin \bar{\ell} \sin \bar{\psi}) \mathrm{d} \tau+2 \bar{\ell} t^{*}+u t^{*} \sin \bar{\ell}
\end{aligned}
$$

If $\bar{\ell}$ is sufficiently small $(\bar{\ell} \rightarrow 0), 1-\cos \bar{\ell}+\sin \bar{\ell} \sin \bar{\ell} \rightarrow 0,2 \bar{\ell} \rightarrow 0$ and $u \sin \bar{\ell} \rightarrow 0$. For a given finite $t^{*}, \ell_{3} \triangleq y_{3}-x_{r}$ is bounded and $\left\|\ell_{3}\right\| \ll\left\|x_{r}\right\|$. The "measurement noise" of $x_{v}$ is caused by the accumulation of $\ell_{1}$ and $\ell_{2}$ over time. In order to reduce the measurement noise accumulation over time, the initial moment should be updated after a short period $t^{*}$. It has been reported that $e$ is in the slow subsystem of the two-time-scale structure. Therefore, $e\left(t_{0}+t^{*}\right)-e\left(t_{0}\right) \rightarrow 0$ if $t^{*}$ is small enough and $y_{3}=x_{r}+\ell_{3}$, where $\ell_{3}$ is regarded as bounded measurement noise. Therefore, we get a structure of two chains of integrators, and all assumptions of Theorem 1 hold. A nonlinear observer for (13) is designed as follows:

$$
\begin{aligned}
& \dot{\hat{\bar{\psi}}}=\hat{r}+\frac{\alpha_{11}}{\varepsilon_{(11)}(t)}\left(y_{1}-\hat{\bar{\psi}}_{1}\right) \\
& \dot{\hat{x}}_{v}=\hat{v}+\frac{\alpha_{21}}{\varepsilon_{(21)}(t)}\left(y_{3}-\hat{x}_{v}\right) \\
& \dot{\hat{v}}=-\frac{\hat{m}_{11}}{\hat{m}_{22}} u \hat{r}-\frac{\hat{d}_{22}}{\hat{m}_{22}} \hat{v}+\hat{x}_{5}+\frac{\alpha_{22}}{\varepsilon_{(22)}^{2}(t)}\left(y_{3}-\hat{x}_{v}\right) \\
& \dot{\hat{r}}=\frac{\hat{m}_{11}-\hat{m}_{22}}{\hat{m}_{33}} u \hat{v}-\frac{\hat{d}_{33}}{\hat{m}_{33}} \hat{r}+\hat{x}_{6}+\frac{\alpha_{12}}{\varepsilon_{(12)}^{2}(t)}\left(y_{1}-\hat{\bar{\psi}}\right) \\
& \dot{\hat{x}}_{5}=\frac{\alpha_{23}}{\varepsilon_{(23)}^{3}(t)}\left(y_{3}-\hat{x}_{v}\right) \\
& \dot{\hat{x}}_{6}=\frac{\alpha_{13}}{\varepsilon_{(13)}^{3}(t)}\left(y_{1}-\hat{\bar{\psi}}\right)
\end{aligned}
$$

where $\varepsilon_{(i j)}$ S are determined by $10 . \quad \hat{m}_{i i}$ and $\hat{d}_{i i}$ are identification values of $m_{i i}$ and $d_{i i}$ respectively, which tolerate identification errors divergent from the true values. According to Theorem 1 and Lemma 1 , as a result of the state feedback law $\delta=\mathcal{Y}(\bar{\psi}, e, v, r)$ in [13] making the system (13) without $\tau_{1}$ and $\tau_{2}$ globally exponentially stable, trajectories of state- and output-feedback $(\delta=\mathcal{Y}(\hat{\bar{\psi}}, e, \hat{v}, \hat{r})$ based on the proposed observer $)$ are close enough in presence of environmental disturbances. To illustrate the result intuitively, numerical simulations of the path following example are given. A monohull ship, borrowed from [24], is utilized to evaluate the performance of the proposed observer. The parameters are given in Appendix D, 
In simulations, we use the state feedback law and some results in [13] and pay more attention to the observer performance. The control law based on back-stepping method makes states converge into an invariant set asymptotically if $\tau_{1}=0$ and $\left|\tau_{2}\right| \leq \gamma_{0}+\gamma_{v}|v|+\gamma_{r}|r|$. The surge velocity $u$ is selected as $7 \mathrm{~m} / \mathrm{s}$. The controller parameters are selected according to the rule in [13], and controller parameters, initial values of system states, and initial values of observers are given in Table III. Define $\hat{a}_{11}=-\hat{m}_{11} / \hat{m}_{22}, \hat{a}_{12}=-\hat{d}_{22} / \hat{m}_{22}, \hat{a}_{21}=\left(\hat{m}_{11}-\right.$ $\left.\hat{m}_{22}\right) / \hat{m}_{33}$, and $\hat{a}_{22}=-\hat{d}_{33} / \hat{m}_{33}$. Coefficients of the observer are also selected as $\alpha_{11}=22, \alpha_{12}=$ 41, $\alpha_{13}=20, \alpha_{21}=22, \alpha_{22}=41$ and $\alpha_{23}=20$. The simulations are conducted in Simulink, and the measurement noise $v$ is generated by the block, Uniform Random Number, where the signals are limited to $[-0.001,0.001]$ and the sample times both are 0.01 seconds for $y_{1}$ and $y_{2}$. The environmental disturbances are selected as $\tau_{1}=0$ and $\tau_{2} \leq 0.005$, which is generated by the combination of two blocks, Uniform Random Number and Sine Wave.

Table IV] gives the parameters of all the cases. To investigate the effects of identification errors, parameters $\hat{m}_{i i}$ and $\hat{d}_{i i}$ in the observer are selected slightly different and significant different from the real values, respectively. In accordance with the analysis in Section 2, if $\varepsilon_{1}$ and $\varepsilon_{2}$ are chosen equivalently, the nonlinear-gain observer becomes a linear high gain observer. Some results of MIMO systems with linear-gain high gain observers are reported in [14. Firstly, the tradeoff of linear-gain observers between state reconstruction speed and noise attenuation is shown in Fig. 446. It is illustrated that a small $\varepsilon$ leads a quick transient response and amplifies the effect of measurement noise. Since decreasing $\varepsilon$ of the lineargain observer casues the estimation error to decay faster to the invariant set, the observer in Fig. 6 has the fastest convergence speed, whereas it is sensitive to measurement noise. This contrasts with that Fig. 4 has the least effect of measurement noise in the steadystate stage. Simulation results of the proposed updated-gain observer is given in Fig. 7] with $\varepsilon_{1}=0.05, \varepsilon_{2}=0.9, d_{1}=0.92$ and $d_{2}=0.4$. The immunity to measurement noise of the proposed observer is almost the same as Fig. 4, and the reconstruction speed in Fig. 7 is faster than Fig. 4. Besides, Fig. 8 and Fig. 9 illustrate the performance recovery of the proposed observer intuitively, that is, trajectories with state- and output feedback are almost the same. The behavior we see in Fig. 10 is the control input, rudder angles $\delta$, in the both cases. Due to the well-known peaking phenomenon, the case of output feedback is full rudder 275 in the transient stage. At the end, the simulation of the proposed observer is conducted with 
significant parameter errors. In Case 2, the relative identification errors, which are defined as $\Delta_{r}=|x-\hat{x}| /|x| \times 100 \%$, are $25.50 \%, 22.18 \%, 25.93 \%$ and $36.56 \%$. Fig. 11 gives the transient and steady-state responses of the estimation errors in this case. The simulation results of the state trajectories are shown in Fig. 12, which are also nearly the same as Fig. 8, meaning that the observer is robust to the identification parameters of the system model. The results are also given in Table IV concisely.

\section{Conclusion}

We have shown that it is capable to design an extended updated-gain high gain observer to estimate the states of MIMO systems, which are globally exponentially stable with respect to an invariant set. The gains are large in the transient stage, and turn relatively small in the steady-state stage. This property makes the observer adequate to make a tradeoff between state reconstruction speed and suppression of measurement noise. Furthermore, the proposed observer has the ability of performance recovery, and it is robust to the identification errors of system model. Two examples of marine surface vessels, roll stabilization and path following, are introduced to show the effectiveness of the proposed observer. In the roll stabilization example, the updated-gain observer recovers the undetectable states quickly. In the path following example, the robustness and the ability of performance recovery are verified, that is the trajectories of state- and output-feedback are almost indistinguishable. The observer design procedure is uncomplicated. Further work will investigate the approach to overcome some limitations of the proposed observer, including application to non-minimum phase systems, application to systems with unmodeled fast dynamics, and weakening the assumptions of unknown exogenous inputs and measurement noise.

\section{Appendices}

\section{A. Proof of Lemma 1}

Proof. Eq. (3) can be rewritten as: $\dot{\chi}=f_{s}(\chi, 0)+\bar{g}(t, \zeta)$. Suppose that $\dot{\chi}=f_{s}(\chi, 0)$ has a globally exponentially stable equilibrium at the origin. $\dot{\chi}=f_{s}(\chi, 0)+\bar{g}(t, \zeta)$ is regarded as a perturbation of $\dot{\chi}=f_{s}(\chi, 0)$, and $\bar{g}(t, \zeta)=\left[\left(B g_{2}(t, \zeta)\right)^{\top}, g_{1}(t, \zeta)^{\top}, \mathbf{0}\right]^{\top}$. According to the converse Lyapunov theorem of exponential stability, there exists a continuously differentiable function $V(\chi)$ for $\dot{\chi}=f_{s}(\chi, 0)$ satisfying the following inequalities: $c_{1}\|\chi\|^{2} \leq V(\chi) \leq c_{2}\|\chi\|^{2}$; 
$\partial V / \partial \chi \cdot f_{s}(\chi, 0) \leq-c_{3}\|\chi\|^{2} ;\|\partial V / \partial \chi\| \leq c_{4}\|\chi\|$. The derivative of $V(t, \chi)$ along the trajectory of $(3)$ is

$$
\begin{aligned}
\dot{V}(t, \chi) & =\frac{\partial V}{\partial t}+\frac{\partial V}{\partial \chi}\left(f_{s}(\chi, 0)+\bar{g}(t, \zeta)\right) \leq-c_{3}\|\chi\|^{2}+\left\|\frac{\partial V}{\partial \chi}\right\| \cdot\|\bar{g}(t, \zeta)\| \\
& =-(1-\theta) c_{3}\|\chi\|-\theta c_{3}\|\chi\|^{2}+c_{4} \bar{g}_{0}\|\chi\| \leq-(1-\theta) c_{3}\|\chi\|^{2}, \quad \forall\|\chi\| \geq \bar{\chi} \\
\dot{V}(t, \chi) & \leq-(1-\theta) c_{3}(d(\chi, \mathcal{I})-\bar{\chi})^{2} \triangleq-\alpha^{(3)}\left(\|\chi\|_{\mathcal{I}}\right), \quad \forall\|\chi\| \geq \bar{\chi}
\end{aligned}
$$

where $\theta \in(0,1)$ and $\bar{\chi}=\bar{g}_{0} c_{4} /\left(\theta c_{3}\right)$. Since $d(\chi, \mathcal{I})-\bar{\chi} \geq 0, \alpha^{(3)}(\cdot)$ is a continuous, positive definite function for $\chi \in \mathbb{R}^{n+m+n_{\theta}} \backslash \mathcal{I}$. It can be summarized as Lemma 1 .

\section{B. Proof of Theorem 1}

Proof. We will proof the theorem in four parts.

B.1. No escape time in $e(t)$

For the purpose of analysis, we define a scaled vector of $e(t)$ as $\eta=\left[\eta_{1 *}^{\top}, \ldots, \eta_{p *}^{\top}\right]^{\top}$ with

$$
\eta_{i *}=\left[\eta_{i 1}, \ldots, \eta_{i n_{i}}\right]^{\top}, \quad \eta_{i j}=\frac{x_{i j}-\hat{x}_{i j}}{\varepsilon_{(i 1)}(t)^{n_{i}+1-j}}, 1 \leq i \leq p, 1 \leq j \leq n_{i}
$$

Define

$$
\begin{aligned}
& D(\varepsilon(t))=\operatorname{blockdiag}\left(D_{1}(\varepsilon(t)), \ldots, D_{p}(\varepsilon(t))\right) \\
& D_{i}(\varepsilon(t))=\operatorname{diag}\left[\frac{1}{\varepsilon_{(i 1)}(t)^{n_{k}}}, \ldots, \frac{1}{\varepsilon_{(i 1)}(t)}\right], 1 \leq i \leq p
\end{aligned}
$$

and $\varepsilon(t)=\left[\varepsilon_{(11)}, \ldots, \varepsilon_{(p 1)}\right]^{\top}$. Thus, $\eta=D(\varepsilon(t)) e$.

Remark 5. For a given $i$ (w.r.t. one chain of integrators), only $\varepsilon_{(i 1)}$ is utilized in scaled functions instead of $\varepsilon_{(i 1)}, \ldots, \varepsilon_{(i p)}$. According to Assumption $3, \varepsilon_{f}$ is a small positive constant and $\varepsilon_{f} \ll 1$.

$$
\varepsilon_{i 1}(t)=\left[\frac{1}{\varepsilon_{1}}+d_{i}\left(\frac{1}{\varepsilon_{2}}-\frac{1}{\varepsilon_{1}}\right) \cdot \frac{\operatorname{sat}\left(\frac{(y-C \hat{x})_{i}}{d_{i}}\right)}{(y-C \hat{x})_{i}}\right]^{-1}
$$

If $(y-C \hat{x})_{i} \in\left[-d_{i}, d_{i}\right], \varepsilon_{i 1}(t)=\varepsilon_{i j}(t)\left(j=2, \ldots, n_{i}\right)$; otherwise,

$$
\varepsilon_{(i 1)}(t)=\varepsilon_{1}\left[1+\mathcal{O}\left(\varepsilon_{f}\right)-\frac{d_{i}}{(y-C \hat{x})_{i}}\right]^{-1}, \varepsilon_{(i j)}(t)=\varepsilon_{1}\left[1+\mathcal{O}\left(\varepsilon_{f}^{j}\right)-\frac{d_{i}}{(y-C \hat{x})_{i}}\right]^{-1 / j}
$$

315

There exists a positive constant $d_{i}^{*}$ such that $\left|\varepsilon_{(i 1)}(t)-\varepsilon_{(i j)}(t)\right|<\gamma, \forall\left(\gamma,(y-C \hat{x})_{i}\right) \in\left(0, \varepsilon_{2}\right) \times$ $\left\{\mathbb{R} \backslash\left[-d_{i}^{*}, d_{i}\right] \cup\left[d_{i}, d_{i}^{*}\right]\right\}$. Therefore, the scaled vector $\eta(t)$ is reasonable. 
Notice that the observer dynamics can be rewritten as

$$
\dot{\hat{x}}=A \hat{x}+B[\hat{\psi}(\hat{x})+\hat{\Sigma}]+\mathcal{H}(\varepsilon)(y-C \hat{x}),
$$

where $\mathcal{H}(\varepsilon)$ denotes a nonlinear vector field of $\varepsilon_{i j}$ and $t$. Let $\bar{\chi}(t)=\operatorname{col}(\chi, \hat{x}, \varepsilon)$, and $\dot{\bar{\chi}}=$ $f_{a}(t, \chi, \hat{x}, \varepsilon)$. Denote $F(x, z)=\psi(x, z)-\hat{\psi}(\hat{x})+g_{2}(t, \zeta)$. Hence, the extended vector $\hat{\Sigma}$ is used to estimate $F(x, z)$, including the identification errors, unmodeled dynamics and environmental disturbances.

$$
\mathcal{L}_{f_{a}} e=A e+B[F(\cdot)-\hat{\Sigma}(\cdot)]-\mathcal{H}(\varepsilon)(C e+\ell)
$$

According to (15), it yields

$$
\mathcal{L}_{f_{a}} \eta_{i j}=\frac{1}{\varepsilon_{(i 1)}^{n_{i}+1-j}} \cdot \mathcal{L}_{f_{a}} e_{i j}-\frac{\mathcal{L}_{f_{a}} \varepsilon_{(i 1)}}{\varepsilon_{(i 1)}} \cdot \eta_{i j}\left(n_{i}+1-j\right)
$$

Combining (18) and (19), we get

$$
\varepsilon_{(i 1)} \mathcal{L}_{f_{a}} \eta_{i *}=\left(A_{i}-\mathcal{H}_{i}^{*} C_{i}-R_{i} \mathcal{L}_{f_{a}} \varepsilon_{(i 1)}\right) \cdot \eta_{i *}+\tilde{\Delta}_{i}(\cdot)+\varepsilon_{(i 1)} \Theta_{i} \mathcal{H}_{i} \ell_{i}
$$

where $R_{i}=\operatorname{diag}\left[n_{i}, \ldots, 1\right], \tilde{\Delta}_{i}=\varepsilon_{(i 1)} \Theta B\left[F_{i}(\cdot)-\hat{\sigma}_{i}(\cdot)\right], \Theta=\operatorname{diag}\left(1 / \varepsilon_{(i 1)}^{n_{i}}, \ldots, 1 / \varepsilon_{(i 1)}^{1}\right), \mathcal{H}_{i}=$ 325 $\left[\alpha_{i, 1} / \varepsilon_{(i 1)}, \ldots, \alpha_{i, n_{i}} / \varepsilon_{\left(i n_{i}\right)}^{n_{i}}\right]^{\top}, \mathcal{H}_{i}^{*}=\left[\alpha_{i, 1}, \alpha_{1,2}^{*}, \ldots, \alpha_{1, n}^{*}\right]^{\top}$ and $\alpha_{i, j}^{*}=\alpha_{i, j}\left(\varepsilon_{(i j)} / \varepsilon_{(i 1)}\right)^{j}$. The error vector of the extended term is denoted as $\Delta=F_{i}(\cdot)-\hat{\Sigma}(\cdot)$, satisfying

$$
\varepsilon_{(i 1)} \dot{\Delta}_{i}(t)=\varepsilon_{(i 1)} \dot{F}_{i}(\cdot)-\varepsilon_{(i 1)} \alpha_{i, n_{i}+1}^{*} \eta_{i 1}-\varepsilon_{(i 1)} \frac{\alpha_{i, n_{i}+1}}{\varepsilon_{\left(i, n_{i}+1\right)}^{n_{i}+1}} \ell_{i}
$$

Define an extended vector as $\bar{\eta}=\left[\bar{\eta}_{1}, \ldots, \bar{\eta}_{p}\right]^{\top}$, where $\bar{\eta}_{i}=\left[\eta_{i}^{* \top}, \Delta_{i}\right]^{\top}$. Therefore,

$$
\varepsilon_{(i 1)} \dot{\bar{\eta}}_{i}=\mathcal{A}_{i}(t) \bar{\eta}_{i}+\mathcal{E}_{i} \dot{F}_{i}+\mathcal{R}_{i} \mathcal{L}_{f_{a}} \varepsilon_{(i 1)} \bar{\eta}_{i}+\mathcal{G}_{i}(\cdot) \ell_{i}
$$

where $\mathcal{R}_{i}=\operatorname{diag}\left(n_{i}, \ldots, 1,0\right)$,

$$
\mathcal{A}_{i}(t)=\left[\begin{array}{cccc}
-\alpha_{i, 1} & 1 & & \\
\vdots & & \ddots & \\
-\alpha_{i, n_{i}}^{*} & & & 1 \\
-\alpha_{i, n_{i}+1}^{*} & 0 & \ldots & 0
\end{array}\right], \mathcal{E}_{i}=\left[\begin{array}{ll}
\mathbf{0}_{n_{i} \times n_{o}} & \\
& \varepsilon_{(i 1)}
\end{array}\right], \mathcal{G}_{i}=\varepsilon_{(i 1)}\left[\begin{array}{ll}
\Theta_{i} \mathcal{H}_{i} & \\
& \frac{\alpha_{i, n_{i}+1}}{\varepsilon_{\left(i, n_{i}+1\right)}^{n_{i}+1}}
\end{array}\right]
$$

In the above analysis, $\mathcal{L}_{f_{a}} \varepsilon_{(i 1)}=0, \forall(y-C x)_{i} \in\left(-d_{i}, d_{i}\right)$; when $(y-C x)_{i}>d_{i}$, the following inequality is a.e. satisfied.

$$
\left\|\mathcal{L}_{f_{a}} \varepsilon_{(i 1)}\right\| \leq \frac{\varepsilon_{1}\left(1-\varepsilon_{f}\right)}{d_{i}} \cdot\left\|(\overbrace{y-C \hat{x}})_{i}\right\| \triangleq h_{i}\left\|(\overbrace{y-C \hat{x}})_{i}\right\| \leq h_{i}\left(\left\|x_{i 2}-\hat{x}_{i 2}\right\|+\left\|\dot{\ell}^{-}\right\|\right)
$$


According to 22 ,

$$
\varepsilon_{(1 i)} \bar{\eta}_{i} \leq\left(A_{i}^{*}+\Delta_{A_{i}}\right) \bar{\eta}_{i}+\varepsilon_{(i 1)}\|\dot{F}\|+n_{i} h_{i}\|\dot{\ell}\|+\mathcal{O}\left(\varepsilon_{(1 i)}^{n_{i}-1}\right)+\|\mathcal{G}\| \bar{\ell}
$$

where $\Delta_{A_{i}}=\mathcal{A}_{i}-A_{i}^{*}$ and

$$
A_{i}^{*}=\left[\begin{array}{cccc}
-\alpha_{i, 1} & 1 & & \\
\vdots & & \ddots & \\
-\alpha_{i, n_{i}} & & & 1 \\
-\alpha_{i, n_{i}+1} & 0 & \ldots & 0
\end{array}\right] .
$$

It has been shown in Remark 5 that $\left\|\Delta_{A_{i}}\right\|$ is bounded. The vector $\ell$ is bounded and $\ell \in \mathcal{C}^{0}$, yielding $\left\|\dot{\ell}^{-}\right\|$is bounded. Matrix $A_{i}^{*}$ is Hurwitz, and there exists a positively definite matrix $P_{i}$ such that $P_{i}^{\top} A_{i}^{*}+A_{i}^{* \top} P_{i}=-I$. Define a Lyapunov function $V_{i}=\bar{\eta}_{i}^{\top} P_{i} \bar{\eta}_{i}$ for 22 ignoring the term $\mathcal{O}\left(\varepsilon_{(i 1)}^{n_{i}-1}\right)$, and

$$
\varepsilon_{(i 1)}^{n_{i}-1} \dot{V}_{i} \leq-\frac{V_{i}}{\lambda_{\max }\left(P_{i}\right)}+2 \frac{\lambda_{\max }\left(P_{i}\right)}{\lambda_{\min }\left(P_{i}\right)}\left\|\Delta_{A_{i}}\right\| \cdot V_{i}+2\|\bar{\eta}\| \lambda_{\max }\left(P_{i}\right)\left[\varepsilon_{(i 1)}\left\|\dot{F}_{i}\right\|+n_{i} h_{i}\left\|\dot{\ell}_{i}\right\|+\|\mathcal{G}\| \bar{\ell}\right]
$$

In summary, the result indicates that $V_{i}$, cannot grow faster than exponentially. Thus $t \mapsto e(t)$ of the given system has no finite escape time in $e$. In the next subsection, the ultimate bound of $e(t)$ will be studied.

\section{B.2. Convergence and boundness of $\bar{\eta}$}

This subsection is inspired by [7], in which a proof of the version of SISO systems without extended terms in [7] is given. For $i=1, \ldots, p$, define a new scaled error vector $\mu$ as $\mu=\left(\mu_{1,1}, \ldots, \mu_{1, n_{1}}, \ldots, \mu_{p, 1}, \ldots, \mu_{p, n_{p}}\right)^{\top}$, where $\mu_{i, j}=\varepsilon_{1}^{j-1} e_{i, j}=\varepsilon_{1}^{j-1}\left(x_{i, j}-\hat{x}_{i, j}\right)\left(j=1, \ldots, n_{i}\right)$, and $\mu_{i, j}=\varepsilon_{1}^{n_{i}}\left(\psi_{i}(\cdot)-\hat{\psi}_{i}(\cdot)+g_{i}(\cdot)-\hat{\sigma}_{i}\right)\left(j=n_{i}+1\right)$. It follows that

$$
\varepsilon_{1} \dot{\mu}=A^{*} \mu+B^{*} \ell+\bar{H}^{*} \bar{\delta}+E^{*} \bar{B} \dot{F}
$$

where $A^{*}=\operatorname{blockdiag}\left(A_{1}^{*}, \ldots, A_{p}^{*}\right), B^{*}=\operatorname{blockdiag}\left(B_{1}^{*}, \ldots, B_{p}^{*}\right), \bar{H}^{*}=\operatorname{blockdiag}\left(\bar{h}_{1}^{*}, \ldots, \bar{h}_{p}^{*}\right)$, $\bar{\delta}=\left[\bar{\delta}_{1}, \ldots, \bar{\delta}_{p}\right]^{\top}, E^{*}=\operatorname{blockdiag}\left(E_{1}^{*}, \ldots, E_{p}^{*}\right)$, and $\bar{B}=\operatorname{blockdiag}\left(\bar{B}_{1}, \ldots, \bar{B}_{p}\right)$ with $E_{i}^{*}=$ $\operatorname{diag}\left(0, \ldots, 0, \varepsilon_{1}^{n_{i}+1}\right), \bar{B}_{i}=(0, \ldots, 0,1)^{\top}, B_{i}^{*}=\left(-\alpha_{i, 1}, \ldots, \alpha_{i, n_{i}}, \alpha_{i, n_{i}+1}\right)^{\top}, \bar{h}_{i}^{*}=\left(\bar{h}_{i 1}, \ldots, \bar{h}_{i n_{i}}\right)^{\top}$, and

$$
\bar{h}_{i j}=\alpha_{i, j}\left[1-\left(\frac{\varepsilon_{1}}{\varepsilon_{2}}\right)^{j}\right], \bar{\delta}_{i}=\operatorname{sat}\left(\frac{e_{i 1}+\ell_{i}}{d_{i}}\right) d_{i}
$$


Assumption 3 implies that each characteristic equation has a fast root. Without loss $Y=\operatorname{blockdiag}\left(Y_{1}, \ldots, Y_{p}\right)$ and $Z=\operatorname{blockdiag}\left(Z_{1}, \ldots, Z_{p}\right)$ with

$$
Y_{i}=\left[\begin{array}{ccccc}
-\beta_{i, 1} & 1 & \ldots & \ldots & 0 \\
-\beta_{i, 2} & 0 & 1 & \ldots & 0 \\
\vdots & & & \ddots & \vdots \\
-\beta_{i, n_{i}} & 0 & \ldots & 0 & 1
\end{array}\right], Z_{i}=\left[\begin{array}{lllll}
1 & 0 & \ldots & \ldots & 0
\end{array}\right]_{1 \times\left(n_{i}+1\right)}
$$

The inverse of $T$ is $T^{-1}=[M, N]$, where the columns of $M$ and $N$ are the bases of $\mathcal{N}\left(A^{*}\right)$ and $\mathcal{R}\left(A^{*}\right)$, respectively. In the new coordinate, we get

$$
\begin{aligned}
& \varepsilon_{1} \dot{\Upsilon}=Y A_{(2)}^{*} M \Upsilon+Y B_{(2)}^{*} \ell+Y \bar{H}^{*} \bar{\delta}+Y E^{*} \bar{B} \dot{F} \\
& \varepsilon_{1} \dot{\mu}^{*}=-\lambda \mu^{*}+\Upsilon_{(1)}-\Pi \ell-\Pi\left(1-\frac{\varepsilon_{1}}{\varepsilon_{2}}\right) \bar{\delta}
\end{aligned}
$$

where

$$
\Upsilon_{(1)}=\left[\begin{array}{c}
\Upsilon_{1,1} \\
\vdots \\
\Upsilon_{p, 1}
\end{array}\right], \Pi=\left[\begin{array}{ccc}
\lambda+\beta_{1,1} & & \\
& \ddots & \\
& & \lambda+\beta_{p, 1}
\end{array}\right]
$$

The term $Y A_{(2)}^{*} M$ in $(28)$ is Hurwitz, and there exists a positive definite matrix $Q$ such that $Q^{\top} Y A_{(2)}^{*}+\left(Y A_{(2)}^{*}\right)^{\top} Q=-I$. The proof outline is given in Appendix $\mathrm{C}$. Define a 365 Lyapunov function candidate for $(28)$ as $W_{1}=\Upsilon^{\top} Q \Upsilon$. Let $L_{Q}=\left\|Q Y B_{(2)}^{*}\right\| \bar{\ell}+\left\|Q Y \bar{H}^{*}\right\| d^{*}+$ $\left\|Q Y E^{*} \bar{B}\right\|\|\dot{F}\|$, and $d^{*}=\max \left\{d_{1}, \ldots, d_{p}\right\}$. It is obvious that $L_{Q}$ is a positive constant if $\varepsilon_{1}$ 
and $\varepsilon_{2}$ are given.

$$
\dot{W}_{1}=\frac{1}{\varepsilon_{1}}\left(-\Upsilon^{\top} \Upsilon+2 \Upsilon^{\top} Q Y\left(B_{(2)}^{*} \bar{\ell}+\bar{h} \bar{\delta}+\bar{B} \dot{F}\right)\right) \leq-\frac{1}{\varepsilon_{1}}\|\Upsilon\|^{2}+\frac{2}{\varepsilon_{1}}\|\Upsilon\| \cdot L_{Q}
$$

It yields that

$$
\dot{W}_{1} \leq-\frac{1}{2 \varepsilon_{1} \lambda_{\max }(Q)} W_{1}, \forall W_{1} \leq L_{W}
$$

for

$$
L_{W}=16 \lambda_{\max }(Q)\left(\left\|Q Y B_{(2)}^{*}\right\| \bar{\ell}+\left\|Q Y \bar{H}^{*}\right\| d^{*}+\left\|Q Y E^{*} \bar{B}\right\|\|\dot{F}\|\right)^{2}
$$

Denote $\Omega_{1}=\left\{\hat{x} \mid W_{1} \leq L_{W}\right\}$. It shows that $\Upsilon$ is bounded, and the output estimation error trajectories come into the invariant set $\Omega_{1}$ within the interval $\left[t_{0}, t_{0}+T_{1}(\varepsilon)\right]$, for

$$
T_{1}\left(\varepsilon_{1}\right)=2 \varepsilon_{1} \lambda_{\max }(Q) \ln \left(\frac{\Upsilon^{\top}\left(t_{0}\right) Q \Upsilon\left(t_{0}\right)}{L_{W}}\right)
$$

If $0<\varepsilon_{1} \ll \varepsilon_{2}<1, T_{1}\left(\varepsilon_{1}\right) \rightarrow 0$ as $\varepsilon_{1} \rightarrow 0$. The tighter bound on the state vector $\Upsilon_{(1)}$ is denoted as

$$
\left\|\Upsilon_{(1)}\right\| \leq k_{0}+k_{1} \bar{\ell}+k_{2} \lambda d^{*}+k_{4} d^{*}+k_{5}\|\dot{F}\|
$$

The next step is studying the ultimate bound of $\mu^{*}$. We will prove that the trajectory 375 of $\left(y_{1}-\hat{x}_{11}, \ldots, y_{p}-\hat{x}_{p 1}\right)^{\top}$ comes into the set $\Omega_{2}$ monotonically, where $\Omega_{2}=\left\{\left|y_{1}-\hat{x}_{11}\right|<\right.$ $\left.d_{1}\right\} \times \ldots \times\left\{\left|y_{p}-\hat{x}_{p 1}\right|<d_{p}\right\}$. Define a Lyapunov function candidate for $(29)$ as $W_{1}=\frac{1}{2} \mu^{* \top} \mu^{*}$.

$$
\dot{W}_{2} \leq-\frac{\lambda}{\varepsilon_{1}}\left\|\mu^{*}\right\|^{2}+\left\|\mu^{*}\right\| \cdot\left(\frac{1}{\varepsilon_{1}}\left\|\Upsilon_{(1)}\right\|+\frac{1}{\varepsilon_{1}}\left(\lambda+\beta_{1}^{*}\right) \bar{\ell}+\left(\frac{1}{\varepsilon_{1}}-\frac{1}{\varepsilon_{2}}\right)\left(\lambda+\beta_{1}^{*}\right) d^{*}\right)
$$

where $\beta_{1}^{*}=\max \left\{\beta_{1,1}, \ldots, \beta_{1, p}\right\}, d_{*}=\min \left\{d_{1}, \ldots, d_{p}\right\}$ and $d^{*}=\max \left\{d_{1}, \ldots, d_{p}\right\}$. For $\theta \in$ $(0,1)$

$$
\dot{W}_{2} \leq-\frac{(1-\theta) \lambda}{\varepsilon_{1}}\left\|\mu^{*}\right\|^{2}, \forall\left\|\mu^{*}\right\| \geq U
$$

Denote $\Omega_{3}=\left\{\hat{x} \mid\left\|\mu^{*}\right\| \leq U\right\}$, and

$$
U=\frac{1}{\theta} \bar{\ell}+\frac{1}{\theta} c_{1} d^{*}+\frac{1}{\lambda \theta}\left(k_{0}+c_{2} \bar{\ell}+c_{3} d^{*}+k_{5}\|\dot{F}\|\right)
$$

where $c_{1}=1+k_{2}-\varepsilon_{f}, c_{2}=k_{1}+\beta_{1}^{*}$ and $c_{3}=k_{4}+\beta_{1}^{*}-\varepsilon_{f} \beta_{1}^{*}$. In order to prove the trajectory of $\left(y_{1}-\hat{x}_{11}, \ldots, y_{p}-\hat{x}_{p 1}\right)^{\top}$ will come into the invariant set $\Omega_{2}$, it should guarantee $\left\{\left\|\mu^{*}\right\| \leq U\right\} \subseteq\left\{\left\|\mu^{*}\right\| \leq d_{*}-\bar{\ell}\right\}$. The condition is equivalent to $U \leq d_{*}-\bar{\ell}$. Considering the form of $U$ as (38), the second term should be paid more attention to. Notice $c_{1}=1+k_{2}-\varepsilon_{f}$, and an approach to compute $k_{2}$ is shown in [7] for SISO systems. With a similar procedure, 
$k_{2}$ is given by $k_{2}=\max _{i=1, \ldots, p}\left(1-\left(\varepsilon_{1} / \varepsilon_{2}\right)^{n_{i}-1}\right)$, and denote $n^{*}=\max _{i=1, \ldots, p}\left(n_{i}\right)$. Suppose $d^{*}=k^{*} \cdot d_{*}$ and $k^{*}>1$. If $\bar{\ell} \in\left(0, \bar{\ell}^{*}\right), \lambda \in\left(\lambda^{*},+\infty\right), k \in(0,2)$ and $\varepsilon_{f} \in(0,1 / 2)$, we get Eq. (39). (Parameters $\bar{\ell}^{*}$ and $\lambda^{*}$ will be discussed later.)

$$
U+\bar{\ell} \leq \frac{1}{\theta}\left[(1+\theta) \bar{\ell}+\left(2-\varepsilon_{f}-\varepsilon_{f}^{n^{*}-1}\right) d^{*}+\frac{k_{0}+c_{2} \bar{\ell}^{*}+c_{3} d^{*}+\|\dot{F}\|}{\lambda^{*}}\right]
$$

If we select

$$
\bar{\ell}^{*}=(2-k) \varepsilon_{f}^{n^{*}-1} d^{*} /\left(2-\varepsilon_{f}^{n^{*}-1} k\right), \lambda^{*}=\frac{k_{0}+c_{2} \bar{\ell}+c_{3} d^{*}+k_{5}\|\dot{F}\|}{\varepsilon_{f}^{n-1}\left[(1-k) d^{*}+k \bar{\ell}\right]-2 \bar{\ell}+\varepsilon_{f} d^{*}},
$$

and $\theta=1-k \varepsilon_{f}^{n-1}$, there exists $\varepsilon_{f}^{*} \in(0,1 / 2)$ such that by choosing $\varepsilon_{f} \in\left(0, \varepsilon_{f}^{*}\right)$, the condition $\left\{\left\|\mu^{*}\right\| \leq U\right\} \subseteq\left\{\left\|\mu^{*}\right\| \leq d_{*}-\bar{\ell}\right\}$ is satisfied. According to (37), if $\mu^{*}\left(t_{0}\right)$ is outside $\Omega_{2}, \mu^{*}$ will converge into the invariant set $\Omega_{2}$ within $\left[t_{0}, t_{0}+T_{2}\left(\varepsilon_{1}\right)\right]$, where

$$
T_{2}\left(\varepsilon_{1}\right)=\frac{\varepsilon_{1}}{2 \lambda k \varepsilon_{f}^{n^{*}}} \ln \left(\frac{2 W_{2}\left(\eta_{1}\left(t_{0}\right)\right)}{\left(d_{*}-\bar{\ell}\right)^{2}}\right) \rightarrow 0 \text { as } \varepsilon_{1} \rightarrow 0
$$

After $t_{1}=t_{0}+T_{1}\left(\varepsilon_{1}\right)+T_{2}\left(\varepsilon_{2}\right)$, it is obvious that $\mathcal{L}_{f_{1}} \varepsilon_{i}=0, i=1, \ldots, p$. According to 22 ,

390

$$
\varepsilon_{2} \bar{\eta}=\mathcal{A} \bar{\eta}+\mathcal{E} \dot{F}+\mathcal{G}(\cdot) \ell
$$

In this case, $\Delta_{A_{i}}=\mathbf{0}$ and $\mathcal{A}(t)=A^{*}$. There exists a matrix $S$ satisfying $S \mathcal{A}+\mathcal{A}^{\top} S=-I$. Define a Lyapunov function for 40 as $W_{3}=\bar{\eta}^{\top} S \bar{\eta}$. For $t \in\left[t_{1},+\infty\right)$

$$
\dot{W}_{3}=\frac{1}{\varepsilon_{2}}\left(-\bar{\eta}^{\top} \bar{\eta}+2 \bar{\eta}^{\top} S \mathcal{G} \bar{\ell}+2 \eta^{\top} S \mathcal{E} \dot{F}_{1}\right) \leq-\frac{1}{2 \varepsilon_{2} \lambda_{\max }(S)} W_{3}, \quad \forall W_{3} \geq L_{S}
$$

where $L_{S}=16 \lambda_{\max }(S)(\|S \mathcal{G}\| \bar{\ell}+\|S \mathcal{E}\|\|\dot{F}\|)^{2}$. Denote $\Omega_{4}=\left\{\hat{x} \mid W_{3} \leq L_{S}\right\}$, which is the ultimate bound of $W_{3}(\bar{\eta})$. Suppose $\hat{x}\left(t_{1}\right)$ is outside of $\Omega_{4}$, and the corresponding scaled error vector is $\bar{\eta}\left(t_{1}\right)$. The estimation trajectory will come into $\Omega_{4}$ within the interval $\left[t_{1}, t_{1}+T_{3}\left(\varepsilon_{2}\right)\right]$, where

$$
T_{3}\left(\varepsilon_{2}\right)=2 \varepsilon_{3} \lambda_{\max }(S) \ln \left(\frac{W_{3}\left(\bar{\eta}\left(t_{1}\right)\right)}{L_{S}}\right) \rightarrow 0 \text { as } \varepsilon_{2} \rightarrow 0
$$

The trajectory will come into the positively invariant set $\Omega^{*}=\Omega_{3} \cap \Omega_{4}$ within the time interval $\left[t_{0}, t_{0}+T\left(\varepsilon_{1}, \varepsilon_{2}\right)\right]$, and $T\left(\varepsilon_{1}, \varepsilon_{2}\right)=T_{1}\left(\varepsilon_{1}\right)+T_{2}\left(\varepsilon_{1}\right)+T_{3}\left(\varepsilon_{2}\right) \rightarrow 0$ as $\varepsilon_{2} \rightarrow 0$. The requirement of $\varepsilon_{f}$ results in that $\varepsilon_{2} \rightarrow 0$ makes $\varepsilon_{1} \rightarrow 0$. After the trajectory comes into $\Omega_{4}$, we have

$$
\begin{aligned}
& \max \{\|x(t)-\hat{x}(t)\|,\|\Delta(t)\|\}=\left\|D_{*}^{-1}\left(\varepsilon_{2}\right) \eta(t)\right\| \leq \sqrt{\frac{\lambda_{\max }(S)}{\lambda_{\min }(S)}}\left(4 \frac{\alpha_{n^{*}+1}}{\varepsilon_{2}^{n^{*}}}\|S\| \bar{\ell}+4 \varepsilon_{2}\|S\|\|\dot{F}\|\right) \\
& \triangleq \kappa_{1} \frac{\bar{\ell}}{\varepsilon_{2}^{n^{*}}}+\kappa_{2} \varepsilon_{2} \triangleq F_{r}\left(\varepsilon_{2}, \bar{\ell}\right)
\end{aligned}
$$


The difference between the term $F_{r}\left(\varepsilon_{2}, \bar{\ell}\right)$ and Lemmas in [5] and [7] should be noticed. Such difference is caused by the extended vector $\hat{\Sigma}$. Lemma 2 gives several properties of the function $F_{r}\left(\varepsilon_{2}, \bar{\ell}\right)$. For given measurement noise $\ell(t)$, the ultimate bound $F_{r}\left(\varepsilon_{2}, \bar{\ell}\right)$ gets the minimal

\section{B.3. The boundness of states with output feedback}

The output feedback with the proposed controller after $t_{0}+T\left(\varepsilon_{1}, \varepsilon_{2}\right)$ is $u=\gamma(\theta, \hat{x}+$ $\left.D_{*}^{-1} \eta, \omega, \zeta\right)$, where $D_{*}^{-1}=1 / D(\varepsilon(t))$ for $t \in\left[t_{1}, \infty\right)$. The full system is represented as $\dot{\chi}=$ $f\left(\chi, \zeta, D_{*}^{-1} \bar{\eta}\right)$, where

$$
f=\left[\begin{array}{c}
A x+B\left[\psi\left(x, z, \gamma\left(\theta, \hat{x}+D_{*}^{-1}\left(\varepsilon_{2}\right) \bar{\eta}\right)\right)+g_{2}\right] \\
f_{0}(x, z)+g_{1}(t, \zeta) \\
\Phi\left(\zeta, \hat{x}+D_{*}^{-1}\left(\varepsilon_{2}\right) \bar{\eta}, \zeta\right)
\end{array}\right]
$$

If $\bar{\eta}=0, \dot{\chi}=f(\chi, \zeta, 0)=f_{s}(\chi, \zeta)$ is the autonomous system with state feedback, which is globally uniformly asymptotically stable with respect to a nonempty, closed and invariant set I. According to the converse Lyapunov theorem in [29], there exists a smooth Lyapunov function $V_{s}$ for $\dot{\chi}=f_{s}(\chi, \zeta)$ with respect to $\mathcal{I}$ satisfying (1) there exist two $\mathcal{K}_{\infty}$-functions $\alpha_{1}^{*}(\cdot)$ and $\alpha_{2}^{*}(\cdot)$ for any $\chi \in U_{0}, \alpha_{1}^{*}\left(\|\chi\|_{\mathcal{I}}\right) \leq V_{s}(\chi) \leq \alpha_{2}^{*}\left(\|\chi\|_{\mathcal{I}}\right) ;(2)$ there exists a continuous, positive definite function $\alpha_{3}$ such that for any $\chi \in U_{0} \backslash \mathcal{I}$ and $\zeta \in \mathcal{D}, \mathcal{L}_{f_{s}} V_{s}(\chi) \leq-\alpha_{3}^{*}\left(\|\chi\|_{\mathcal{I}}\right)$. We define a Lyapunov function candidate $V$ for the full system with estimation errors as $V(t, \chi)=V_{s}(t, \chi)$, and

$$
\dot{V} \leq-\alpha_{3}^{*}\left(\|\chi\|_{\mathcal{I}}\right)+\left\|\frac{\partial V}{\partial \chi}\right\|\left\|f\left(\chi, \zeta, D_{*}^{-1}\left(\varepsilon_{2}\right) \eta\right)-f(\chi, \zeta, 0)\right\|
$$

For given $\varepsilon_{2}^{*}>\kappa \bar{\ell}^{1 /\left(n^{*}+1\right)}$ and $\ell_{1}^{*}>0$, if $\varepsilon_{2} \in\left(\kappa \bar{\ell}^{1 /\left(n^{*}+1\right)}, \varepsilon_{2}^{*}\right)$ and $\bar{\ell}<\ell_{1}^{*}$, there exists a positive constant $L_{1}$ such that $\left\|f\left(\chi, \zeta, D_{*}^{-1} \eta\right)-f(\chi, \zeta, 0)\right\| \leq L_{1}\left\|D_{*}^{-1} \eta\right\|=L_{1} F_{r}\left(\varepsilon_{2}, \ell\right)$. We have showed that $\dot{W}_{3}<0$ for all $(\chi, \hat{x}) \in U_{0} \times \partial \Omega^{*}$. Suppose $\max _{\chi \in \Omega^{*}}\|\partial V / \partial \chi\|=L_{2}$. Define $k^{*}=$ $\min _{\chi \in \partial U_{0}} \alpha_{3}^{*}\left(\|\chi\|_{\mathcal{I}}\right) /\left(L_{1} L_{2}\right)$ and $\ell_{2}^{*}=\left(k^{*} / \kappa_{a}\right)^{n *+1}$. If $\bar{\ell} \in\left(0, \ell_{2}^{*}\right), \dot{V}<0$ for all $(\chi, \hat{x}) \in \partial U_{0} \times \Omega^{*}$. Hence $U_{0} \times \Omega^{*}$ is a positively invariant set. If $\chi\left(t_{0}\right) \in U_{0}$ and $\hat{x} \notin \Omega^{*}$, there exists a positive constant $T^{*}$ such that $\chi(t) \in U_{0}, \forall t \in\left[t_{0}, t_{0}+T^{*}\right]$. We hope that the trajectory of $\chi(t)$ is still in $U_{0}$ at $t_{0}+T^{*}$, that is, $\left[t_{0}, t_{0}+T\left(\varepsilon_{1}, \varepsilon_{2}\right)\right) \subset\left[t_{0}, t_{0}+T^{*}\right]$. There exists $\varepsilon_{2}^{(1)}$ such that $0<\varepsilon_{2}<\varepsilon_{2}^{(1)}, T\left(\varepsilon_{1}, \varepsilon_{2}\right) \leq T^{*}$. And there exists $\ell_{3}^{*}$ such that $\kappa\left(\ell_{3}^{*}\right)^{1 /\left(n^{*}-1\right)}<\varepsilon_{2}^{(1)}$. By selecting $\bar{\ell} \in\left(0, \min \left(\ell_{1}^{*}, \ell_{2}^{*}, \ell_{3}^{*}\right)\right]$ and $\varepsilon_{2} \in\left(\kappa_{a} \bar{\ell}^{1 /\left(n^{*}-1\right)}, \min \left(\varepsilon_{M}, \varepsilon_{2}^{(1)}\right)\right)$, the trajectory of $(\chi, \hat{x})$ will come 
into the invariant set $U_{0} \times \Sigma$ in the interval $\left[t_{0}, t_{0}+T\left(\varepsilon_{1}, \varepsilon_{2}\right)\right]$ and $T\left(\varepsilon_{1}, \varepsilon_{2}\right)<T^{*}$. Define $F_{r}^{*} \triangleq\left\|F_{r}\right\|$. According to 44 ,

$$
\dot{V} \leq-\frac{1}{2} \alpha_{3}^{*}(\|\chi\|)_{\mathcal{I}}+L_{1} L_{2} F_{r}^{*}-\frac{1}{2} \alpha_{3}^{*}(\|\chi\|)_{\mathcal{I}} \leq-\frac{1}{2} \alpha_{3}^{*}(\|\chi\|)_{\mathcal{I}}, \quad \forall\|\chi\|_{\mathcal{I}} \geq \chi^{*}
$$

where $\chi^{*}=\alpha_{3}^{*-1}\left(2 L_{1} L_{2} F_{r}^{*}\right)$. We prove that the states of the output feedback system are bounded.

\section{B.4. Performance recovery}

In this subsection, we will explore that the trajectories with state- and output feedback are close enough, namely performance recovery. More details about performance recovery could be found in 30 .

Define $c^{*} \triangleq \sup _{c}\left(\{\chi \mid V(\chi) \leq c\} \subseteq U_{0}\right)$, and the corresponding value of $\|\chi(t)\|_{\mathcal{I}}=c^{(1)}$. There exists a time $T_{4}$ such that $\|\chi(t)\|_{\mathcal{I}} \leq c^{(1)} / 2$ and $\left\|\chi_{r}(t)\right\|_{\mathcal{I}} \leq c^{(1)} / 2$ for $t \geq T_{4}$. Define a vector as $\chi_{e}=\chi-\chi_{r}$. For $t>\min \left(T\left(\varepsilon_{1}, \varepsilon_{2}\right), T_{4}\right)$

$$
\dot{\chi}_{e}=f(\chi, \zeta, 0)-f\left(\chi, \zeta, D_{*}^{-1}\left(\varepsilon_{2}\right) \eta\right)
$$

Define a Lyapunov function for (46) as $V_{2}\left(t, \chi_{e}\right)=V(t, \cdot)$ and it yields $\partial V_{2} / \partial \chi_{e}=\partial V /\left.\partial \chi\right|_{\chi=\chi_{e}}$.

$$
\dot{V}_{2}=\frac{\partial V_{2}}{\partial \chi_{e}}\left(f(\chi, \zeta, 0)-f\left(\chi, \zeta, D_{*}^{-1}\left(\varepsilon_{2}\right) \bar{\eta}\right)\right) \leq-\alpha_{3}^{*}\left(\left\|\chi_{e}\right\|\right)+\left\|\frac{\partial V_{2}}{\partial \chi_{e}}\right\| \cdot L_{1} F_{r}\left(\varepsilon_{2}, \bar{\ell}\right)
$$

It is obvious that $\left\|\chi_{e}\right\| \leq\left\|\chi_{r}\right\|+\|\chi\| \leq c^{(1)}$, and $\chi_{e} \in U_{0}$. Therefore, $\left\|\partial V_{2} / \partial \chi_{e}\right\|$ is bounded for $t>\min \left(T\left(\varepsilon_{1}, \varepsilon_{2}\right), T_{4}\right)$, and the bound is defined as $L_{4}$.

$$
\dot{V}_{2} \leq-\frac{\alpha_{3}^{*}\left(\left\|\chi_{e}\right\|\right)}{2}, \quad \forall\|\chi\|_{\mathcal{I}} \geq \chi_{e}^{*}
$$

where $\chi_{e}^{*}=\alpha_{3}^{*-1}\left(2 L_{1} L_{4} F_{r}^{*}\right)$. We get the ultimate bound of $\left\|\chi(t)-\chi_{r}(t)\right\|$ is $\chi_{e}^{*}$, and $\chi(t)-\chi_{r}(t)$ will come into the ultimate bound in finite time.

\section{Hurwitz characteristic of $Y A_{(2)}^{*} M$}

In this section, we give a proof outline that the matrix $Y A_{(2)}^{*} M$ is Hurwitz. According to the definition of $T^{-1}$, we get $M=\operatorname{blockdiag}\left(M_{1}, \ldots, M_{p}\right)$ and $N=\operatorname{blockdiag}\left(N_{1}, \ldots, N_{p}\right)$, 
where

$$
M_{i}=\left[\begin{array}{cccc}
0 & 0 & \ldots & 0 \\
1 & 0 & \ldots & 0 \\
0 & 1 & \ldots & 0 \\
\vdots & \vdots & \ddots & \vdots \\
0 & 0 & \ldots & 1
\end{array}\right]_{\left(n_{i}+1\right) \times n_{i}} \quad, N_{i}=\left[\begin{array}{c}
1 \\
\beta_{i, 1} \\
\beta_{i, 2} \\
\vdots \\
\beta_{i, n_{i}}
\end{array}\right], i=1, \ldots, p
$$

The polynomials $s^{n_{i}}+\beta_{i, 1} s^{n_{i}}+\ldots+\beta_{i, n_{i}-1} s+\beta_{i, n_{i}}=0$ are Hurwitz for $i=1, \ldots, p$. The forms of $Y, A_{(2)}$ and $M$ are definite, and it is easy to prove that $Y A_{(2)}^{*} M$ is Hurwitz.

\section{Parameters of a marine surface vessel}

This appendix gives the parameters of a monohull ship which is reported in [24]. The yaw moment $u_{2}$ is supposed to be proportional to the rudder angle $\delta$, and $\left(1 / m_{33}\right) u_{2}=b_{2} \delta$. All parameters are in Table II.

Table II: Parameters of the monohull ship

\begin{tabular}{ll|ll}
\hline vessel length & $32 \mathrm{~m}$ & mass & $1.18 \times 10^{5} \mathrm{~kg}$ \\
\hline$m_{11}$ & $1.20 \times 10^{3} \mathrm{~kg}$ & $m_{22}$ & $2.179 \times 10^{5} \mathrm{~kg}$ \\
\hline$m_{33}$ & $6.36 \times 10^{7} \mathrm{~kg} \cdot \mathrm{m}^{2}$ & $d_{11}$ & $2.15 \times 10^{4} \mathrm{~kg} \cdot \mathrm{m}^{-1}$ \\
\hline$d_{22}$ & $1.17 \times 10^{5} \mathrm{~kg} \cdot \mathrm{s}^{-1}$ & $d_{33}$ & $8.02 \times 10^{6} \mathrm{~kg} \cdot \mathrm{m}^{2} \cdot \mathrm{s}^{-1}$ \\
\hline$b_{2}$ & $2.8385 \times 10^{-3}$ & $\max |\delta|$ & $20 \mathrm{deg}$ \\
\hline $\max |\dot{\delta}|$ & $5 \mathrm{deg} / \mathrm{s}$ & \multicolumn{2}{l}{} \\
\hline
\end{tabular}

\section{Acknowledgement}

This paper is partly supported by the National Science Foundation of China $(61473183,61521063$, U1509211), Program of Shanghai Subject Chief Scientist (14XD1402400). The authors would like to thank antonymous reviewers for their constructive suggestions and comments which improve substantially the original manuscript.

\section{References}

[1] G. Besançon, Nonlinear observers and applications, Vol. 363, Springer, 2007.

[2] A. Alessandri, A. Rossi, Increasing-gain observers for nonlinear systems: Stability and design, Automatica 57 (2015) 180-188. doi:10.1016/j.automatica.2015.04.017. 
[6] R. G. Sanfelice, L. Praly, On the performance of high-gain observers with gain adaptation under measurement noise, Automatica 47 (10) (2011) 2165-2176. doi:10.1016/j.automatica.2011.08.002

[7] A. A. Prasov, H. K. Khalil, A nonlinear high-gain observer for systems with measurement noise in a

[8] M. Farza, M. Oueder, R. B. Abdennour, M. M'Saad, High gain observer with updated gain for a class of MIMO nonlinear systems, International Journal of Control 84 (2) (2011) 270-280. doi:10.1080/ 00207179.2010 .550014

[9] M. Oueder, M. Farza, R. B. Abdennour, M. MSaad, A high gain observer with updated gain for a class of MIMO non-triangular systems, Systems \& Control Letters 61 (2) (2012) 298-308. doi:10.1016/j . sysconle.2011.11.009

[10] S. Xiong, W. Wang, X. Liu, Z. Chen, S. Wang, A novel extended state observer, ISA transactions 58 (2015) 309-317. doi:10.1016/j.isatra.2015.07.012

[11] R. Madoński, P. Herman, Survey on methods of increasing the efficiency of extended state disturbance observers, ISA transactions 56 (2015) 18-27. doi:10.1016/j.isatra.2014.11.008

[12] T. I. Fossen, Guidance and control of ocean vehicles, John Wiley \& Sons Inc, 1994.

[13] Z. Li, J. Sun, S. Oh, Design, analysis and experimental validation of a robust nonlinear path following controller for marine surface vessels, Automatica 45 (7) (2009) 1649-1658. doi:10.1016/j . automatica. 2009.03 .010 .

[14] K. Ma, H. K. Khalil, Y. Yao, Guidance law implementation with performance recovery using an extended high-gain observer, Aerospace Science and Technology 24 (1) (2013) 177-186. doi:10.1016/j . ast. 2011. 11.005

[15] M. R. Mokhtari, A. C. Braham, B. Cherki, Extended state observer based control for coaxial-rotor UAV, ISA transactions doi:10.1016/j.isatra.2015.11.024 
[16] A. Gutiérrez-Giles, M. A. Arteaga-Pérez, GPI based velocity/force observer design for robot manipulators, ISA transactions 53 (4) (2014) 929-938. doi:10.1016/j.isatra.2014.03.002.

[17] T. I. Fossen, Handbook of marine craft hydrodynamics and motion control, John Wiley \& Sons, 2011.

[18] J. Du, X. Hu, H. Liu, C. Chen, Adaptive robust output feedback control for a marine dynamic positioning system based on a high-gain observer, Neural Networks and Learning Systems, IEEE Transactions on 26 (11) (2015) 2775 - 2786. doi:10.1109/TNNLS.2015.2396044.

[19] T. Fossen, Å. Grøvlen, et al., Nonlinear output feedback control of dynamically positioned ships using vectorial observer backstepping, Control Systems Technology, IEEE Transactions on 6 (1) (1998) 121-128. doi:10.1109/87.654882

[20] M. Wondergem, E. Lefeber, K. Y. Pettersen, H. Nijmeijer, Output feedback tracking of ships, Control Systems Technology, IEEE Transactions on 19 (2) (2011) 442-448. doi:10.1109/TCST.2010.2045654

[21] D. d. A. Fernandes, A. J. Sørensen, K. Y. Pettersen, D. C. Donha, Output feedback motion control system for observation class ROVs based on a high-gain state observer: Theoretical and experimental results, Control Engineering Practice 39 (2015) 90-102. doi:10.1016/j.conengprac.2014.12.005.

[22] N. Wang, M. Joo Er, Self-constructing adaptive robust fuzzy neural tracking control of surface vehicles with uncertainties and unknown disturbances, Control Systems Technology, IEEE Transactions on 23 (3) (2015) 991-1002. doi:10.1109/TCST.2014.2359880.

[23] R.-Y. Ren, Z.-J. Zou, X.-G. Wang, A two-time scale control law based on singular perturbations used [ in rudder roll stabilization of ships, Ocean Engineering 88 (2014) 488-498. doi:10.1016/j.oceaneng. 2014.07 .006 .

[24] K. D. Do, Z.-P. Jiang, J. Pan, Underactuated ship global tracking under relaxed conditions, Automatic Control, IEEE Transactions on 47 (9) (2002) 1529-1536. doi:10.1109/TAC.2002.802755.

[25] E. Lefeber, K. Y. Pettersen, H. Nijmeijer, Tracking control of an underactuated ship, Control Systems Technology, IEEE Transactions on 11 (1) (2003) 52-61. doi:10.1109/TCST.2002.806465.

[26] G. Zhang, X. Zhang, A novel DVS guidance principle and robust adaptive path-following control for 510 underactuated ships using low frequency gain-learning, ISA transactions 56 (2015) 75-85. doi:10.1016/ j.isatra.2014.12.002.

[27] R. Skjetne, T. Fossen, et al., Nonlinear maneuvering and control of ships, in: OCEANS, 2001. MTS/IEEE Conference and Exhibition, Vol. 3, IEEE, 2001, pp. 1808-1815. doi:10.1109/OCEANS.2001.968121.

[28] P. Kokotovic, H. K. Khalil, J. O'reilly, Singular perturbation methods in control: analysis and design, Vol. 25, SIAM, 1999. 
[29] Y. Lin, E. D. Sontag, Y. Wang, A smooth converse lyapunov theorem for robust stability, SIAM Journal on Control and Optimization 34 (1) (1996) 124-160. doi:10.1137/S0363012993259981.

[30] J. Lei, H. Khalil, Feedback linearization for nonlinear systems with time-varying input and output delays by using high-gain predictors, Automatic Control, IEEE Transactions ondoi:10.1109/TAC.2015.

Table III: Controller parameters and initial values in Example 2

\begin{tabular}{ll|ll}
\hline $\bar{c}_{1}$ & $4.27 \times 10^{-4}$ & $\bar{c}_{2}$ & 0.427 \\
\hline $\bar{c}_{3}$ & 28.175 & $\bar{c}_{4}$ & 0.0705 \\
\hline $\bar{\psi}\left(t_{0}\right)$ & $-30 \mathrm{deg}$ & $e\left(t_{0}\right)$ & $1000 \mathrm{~m}$ \\
\hline$v\left(t_{0}\right)$ & $0 \mathrm{~m} / \mathrm{s}$ & $r\left(t_{0}\right)$ & $0 \mathrm{deg} / \mathrm{s}$ \\
\hline$\hat{\bar{\psi}}\left(t_{0}\right)$ & $0 \mathrm{deg}$ & $\hat{x}_{v}\left(t_{0}\right)$ & $900 \mathrm{~m}$ \\
\hline$\hat{v}\left(t_{0}\right)$ & $0 \mathrm{~m} / \mathrm{s}$ & $\hat{r}\left(t_{0}\right)$ & $0 \mathrm{deg} / \mathrm{s}$ \\
\hline
\end{tabular}


Table IV: Observer parameters and results in Example 2

\begin{tabular}{|c|c|c|c|}
\hline Case 1 & small identification errors & & \\
\hline$\hat{a}_{11}$ & -0.55 & $\hat{a}_{12}$ & -3.9 \\
\hline$\hat{a}_{21}$ & -0.011 & $\hat{a}_{22}$ & -0.127 \\
\hline$\hat{b}_{2}$ & 0.0028358 & & \\
\hline Fig. 4 & $\begin{array}{l}\text { linear gain } \\
\text { slow transient responses }\end{array}$ & $\varepsilon$ & $\begin{array}{l}0.9 \\
\text { small steady-state errors }\end{array}$ \\
\hline Fig. 5 & $\begin{array}{l}\text { linear gain } \\
\text { fast transient responses }\end{array}$ & $\varepsilon$ & $\begin{array}{l}0.1 \\
\text { large steady-state errors }\end{array}$ \\
\hline Fig. 6 & $\begin{array}{l}\text { linear gain } \\
\text { the fastest transient responses }\end{array}$ & $\varepsilon$ & $\begin{array}{l}0.01 \\
\text { steady-state errors cannot be accepted }\end{array}$ \\
\hline Fig. 7 & $\begin{array}{l}\text { updated gain } \\
\text { relatively fast transient responses }\end{array}$ & $\varepsilon_{1}, \varepsilon_{2}$ & $\begin{array}{l}0.05,0.9 \\
\text { small steady-state errors }\end{array}$ \\
\hline Case 2 & large identification errors & & \\
\hline$\hat{a}_{11}$ & -0.4 & $\hat{a}_{12}$ & -3.0 \\
\hline$\hat{a}_{21}$ & -0.008 & $\hat{a}_{22}$ & -0.08 \\
\hline$\hat{b}_{2}$ & 0.0015 & & \\
\hline Fig. 11 & $\begin{array}{l}\text { updated gain } \\
\text { relatively fast transient responses } \\
\text { almost the same as Fig. } 7\end{array}$ & $\varepsilon_{1}, \varepsilon_{2}$ & $\begin{array}{l}0.05,0.9 \\
\text { small steady-state errors } \\
\text { almost the same as Fig. } 7\end{array}$ \\
\hline
\end{tabular}

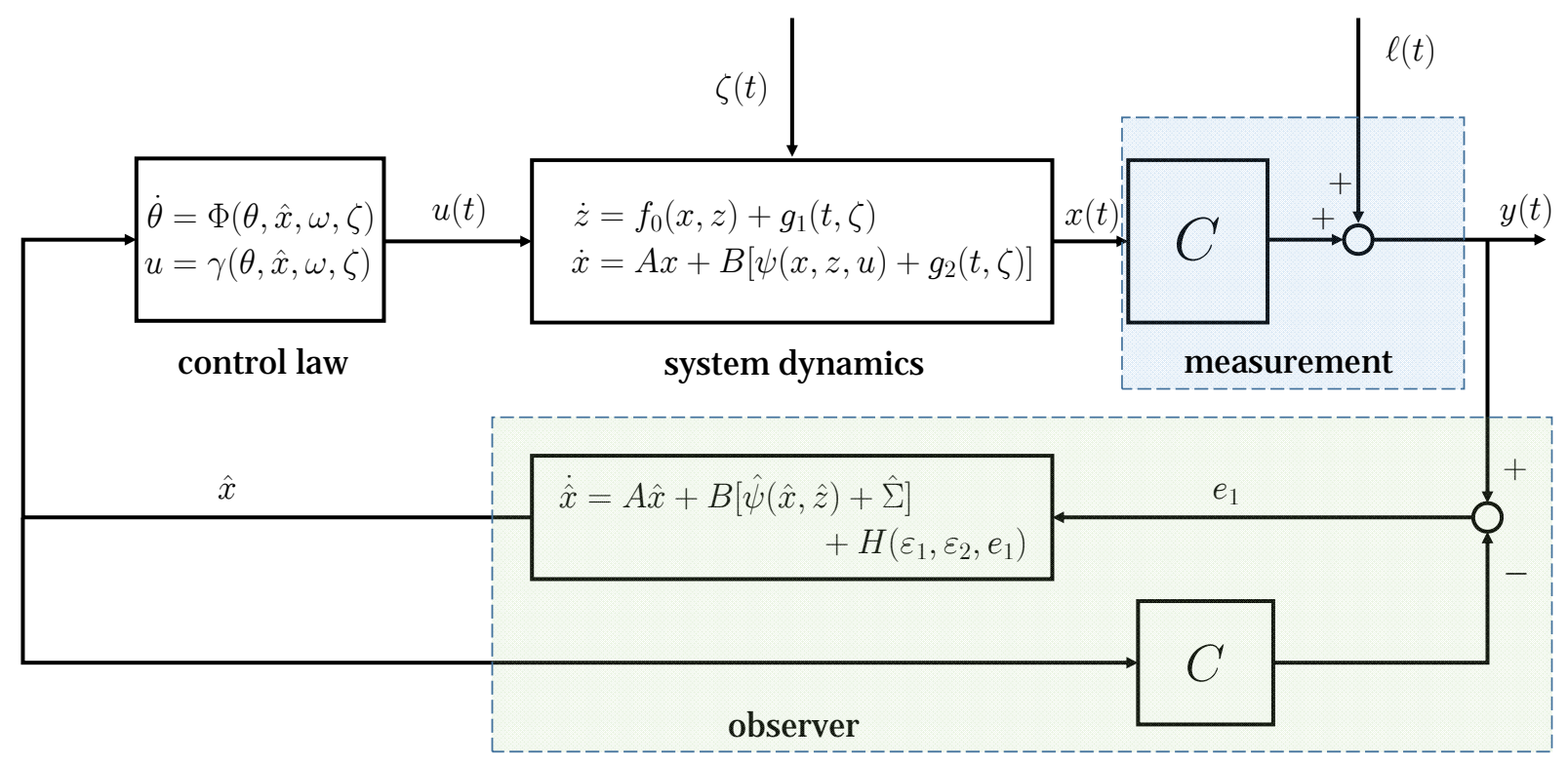

Figure 1: Full system structure with output feedback 


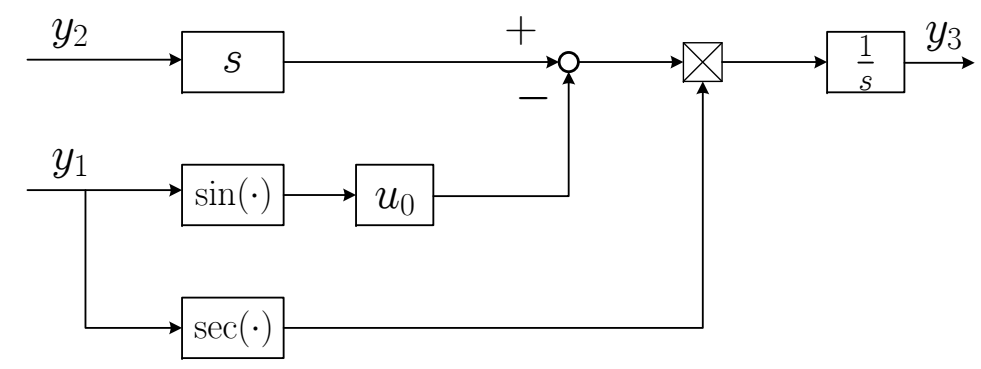

Figure 2: Collection block diagram of $y_{3}$

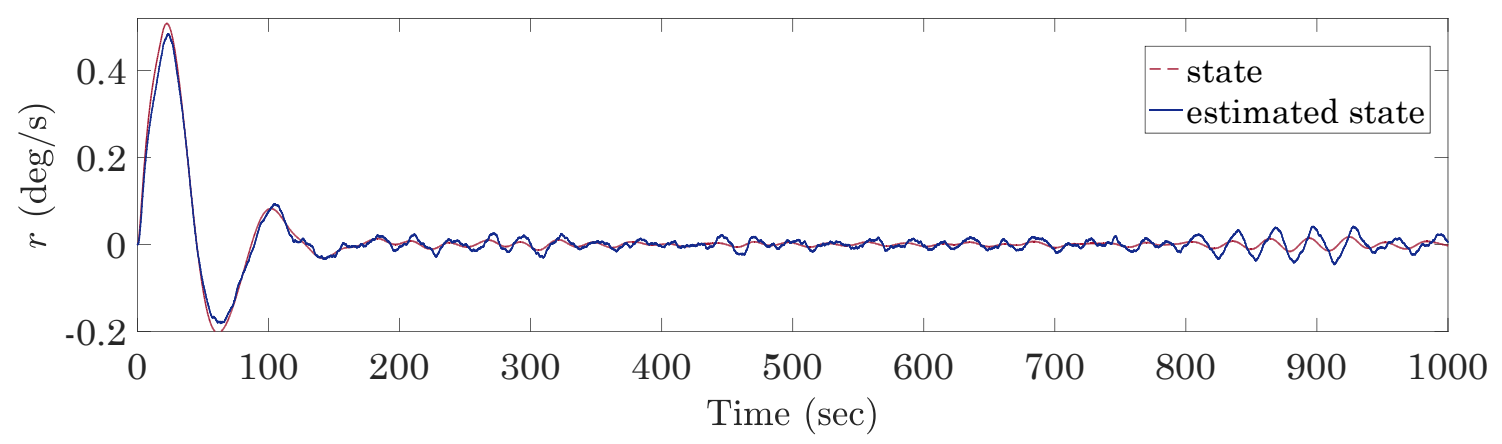

(a)

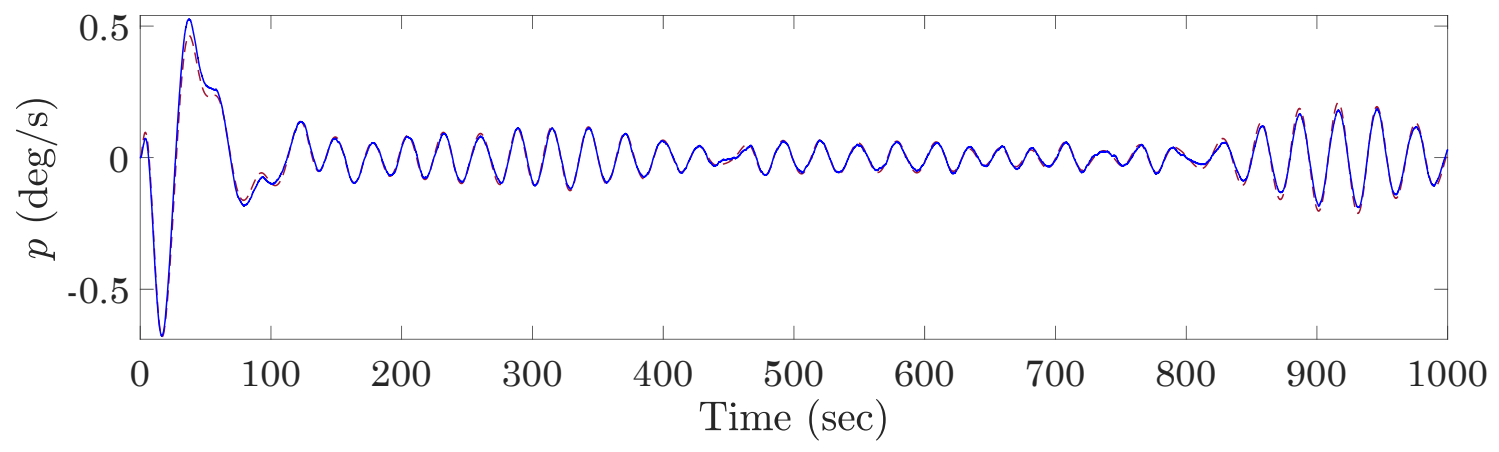

(b)

Figure 3: The plots of unmeasured system states $r, p$ and their estimations $\hat{r}, \hat{p}$ from the proposed observer in Example 1. 


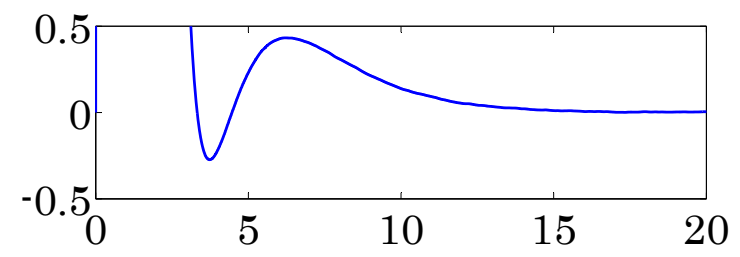

(a)

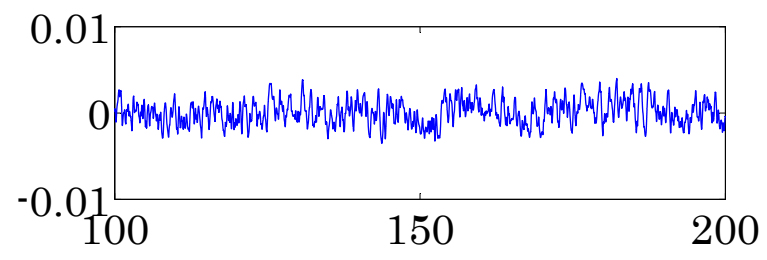

(c)

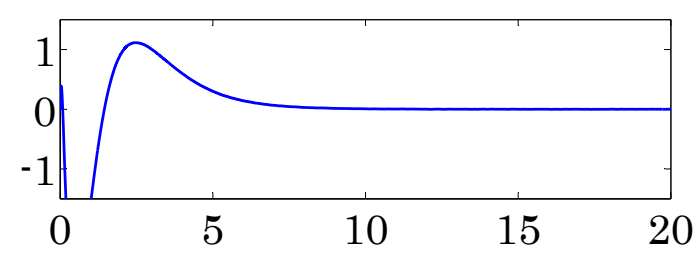

(b)

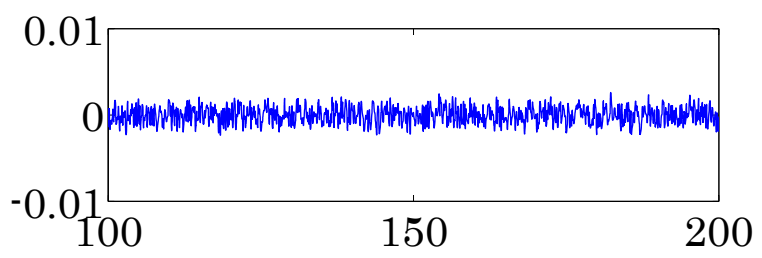

(d)

Time (sec)

Figure 4: Estimation errors of a linear-gain high-gain observer with $\varepsilon_{1}=0.9, \varepsilon_{2}=0.9$ where (a) the transient response of $v-\hat{v}$, (b) the transient response of $r-\hat{r}$, (c) the steady-state response of $v-\hat{v}$ and (d) the steady-state response of $r-\hat{r}$. The unit of $v-\hat{v}$ is $\mathrm{m} / \mathrm{s}$, and the one of $r-\hat{r}$ is $\mathrm{rad} / \mathrm{s}$.

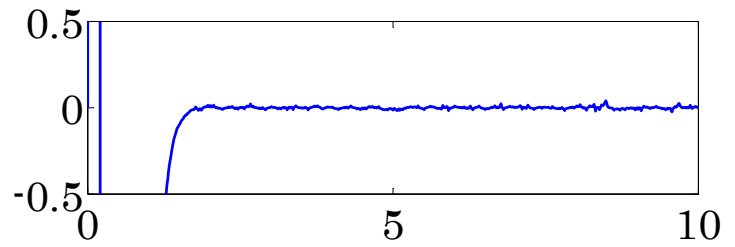

(a)

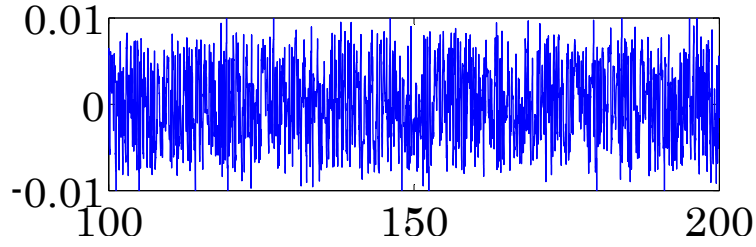

(c)

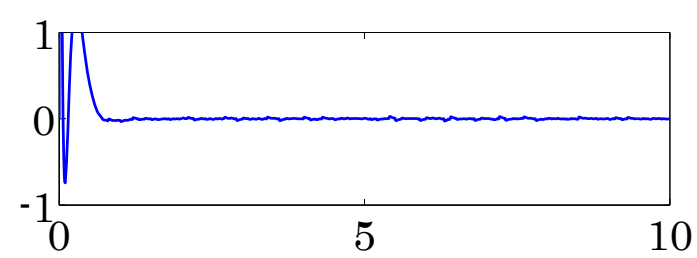

(b)

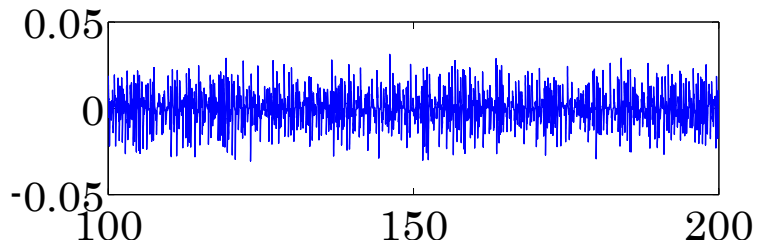

(d)

Time (sec)

Figure 5: Estimation errors of a linear-gain high-gain observer with $\varepsilon_{1}=0.1, \varepsilon_{2}=0.1$ where (a) the transient response of $v-\hat{v}$, (b) the transient response of $r-\hat{r}$, (c) the steady-state response of $v-\hat{v}$ and (d) the steady-state response of $r-\hat{r}$. The unit of $v-\hat{v}$ is $\mathrm{m} / \mathrm{s}$, and the one of $r-\hat{r}$ is $\mathrm{rad} / \mathrm{s}$. 


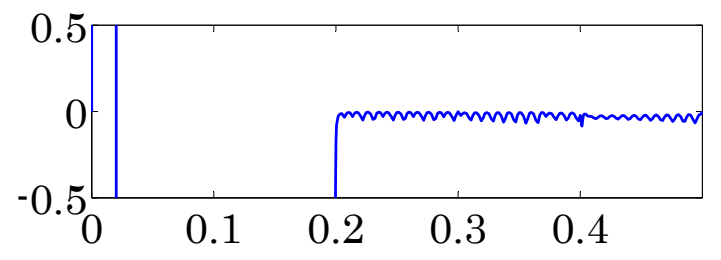

(a)

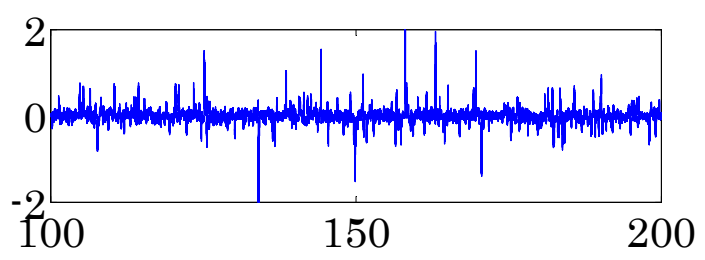

(c)

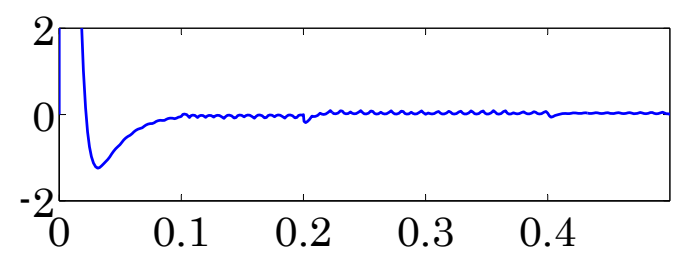

(b)

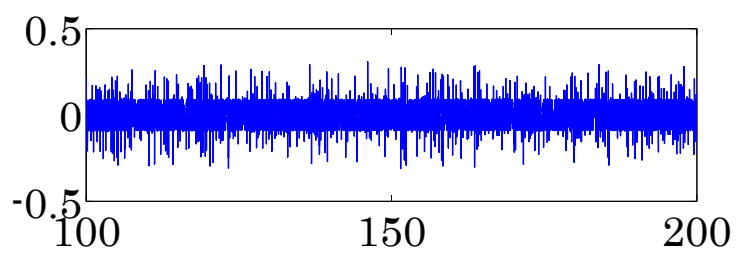

(d)

Time (sec)

Figure 6: Estimation errors of a linear-gain high-gain observer with $\varepsilon_{1}=0.01, \varepsilon_{2}=0.01$ where (a) the transient response of $v-\hat{v}$, (b) the transient response of $r-\hat{r}$, (c) the steady-state response of $v-\hat{v}$ and $(\mathrm{d})$ the steady-state response of $r-\hat{r}$. The unit of $v-\hat{v}$ is $\mathrm{m} / \mathrm{s}$, and the one of $r-\hat{r}$ is $\mathrm{rad} / \mathrm{s}$.

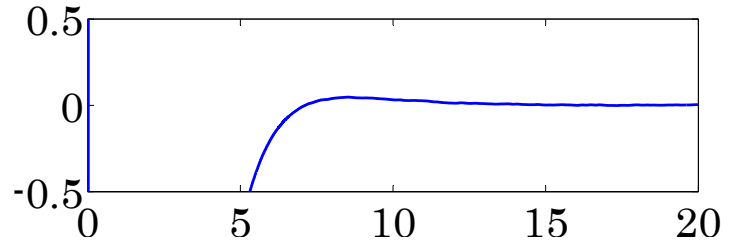

(a)

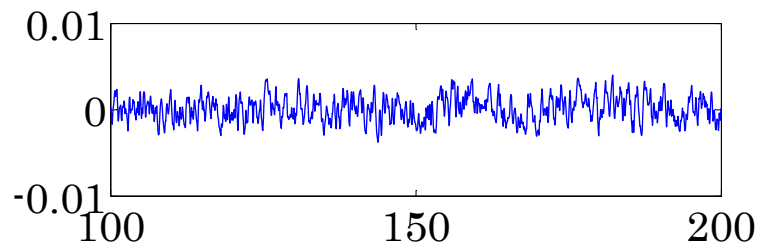

(c)

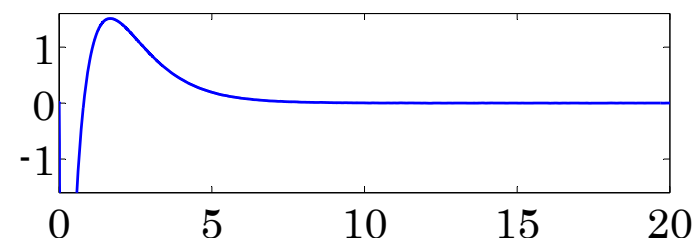

(b)

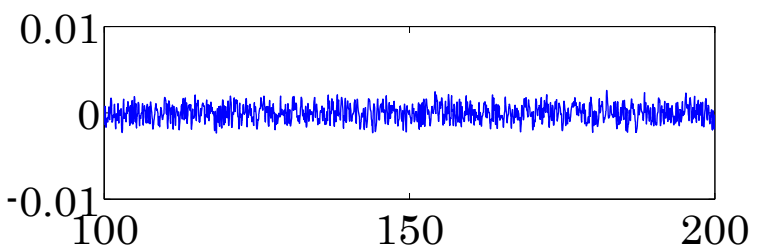

(d)

Time (sec)

Figure 7: Estimation errors of an updated-gain high-gain observer with $\varepsilon_{1}=0.05, \varepsilon_{2}=0.9$ where (a) the transient response of $v-\hat{v}$, (b) the transient response of $r-\hat{r}$, (c) the steady-state response of $v-\hat{v}$ and (d) the steady-state response of $r-\hat{r}$. The unit of $v-\hat{v}$ is $\mathrm{m} / \mathrm{s}$, and the one of $r-\hat{r}$ is $\mathrm{rad} / \mathrm{s}$. 


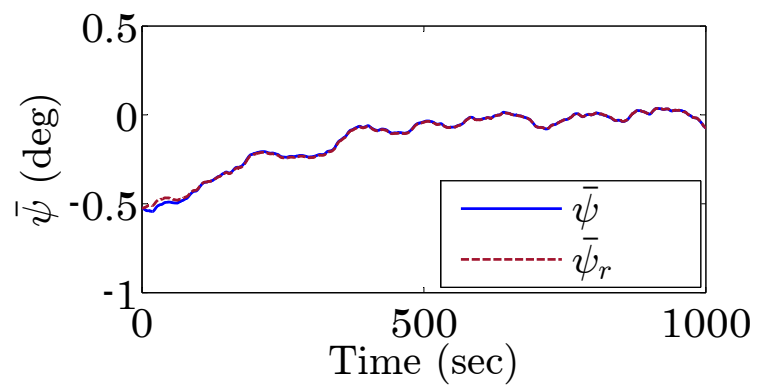

(a)

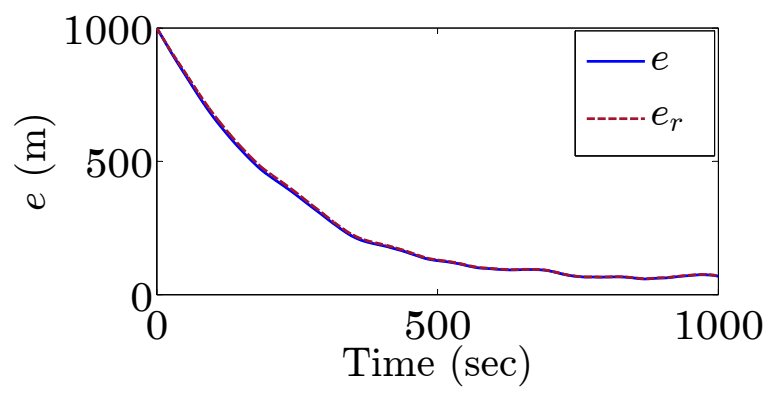

(b)

Figure 8: States with state feedback vs. output feedback where (a) $\bar{\psi}$ and $\bar{\psi}_{r}$, (b) $e$ and $e_{r}$.

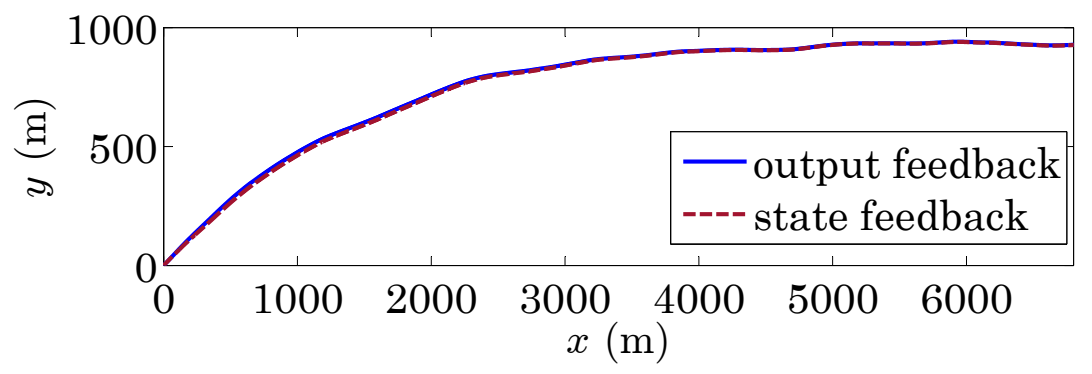

Figure 9: Trajectories with state feedback and output feedback

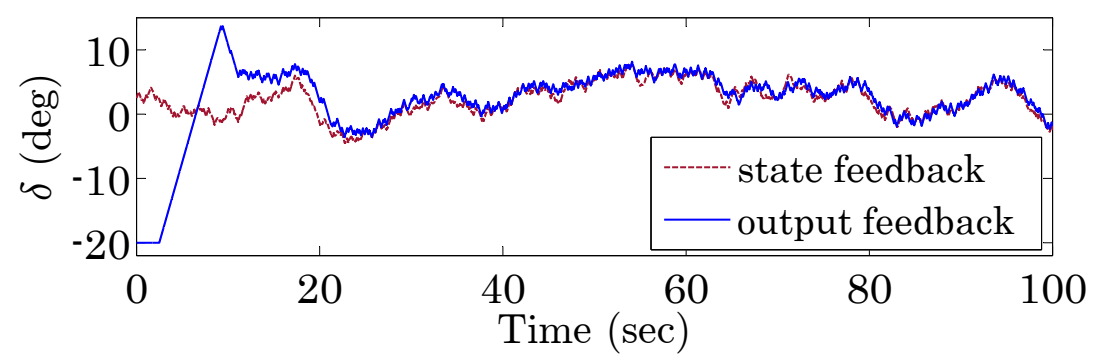

Figure 10: Rudder angle $\delta$ with state feedback and output feedback 


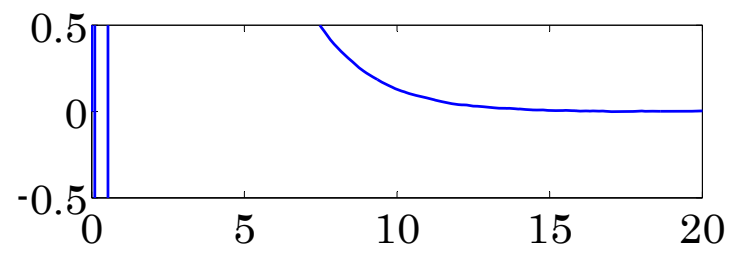

(a)

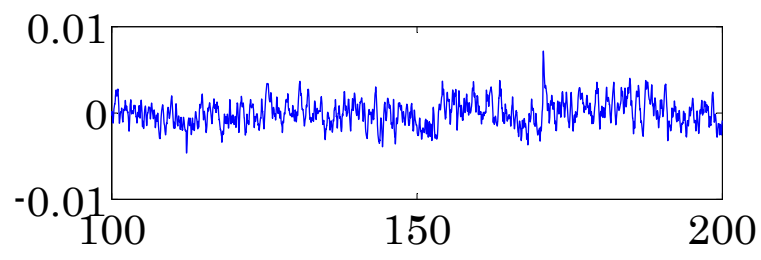

(c)

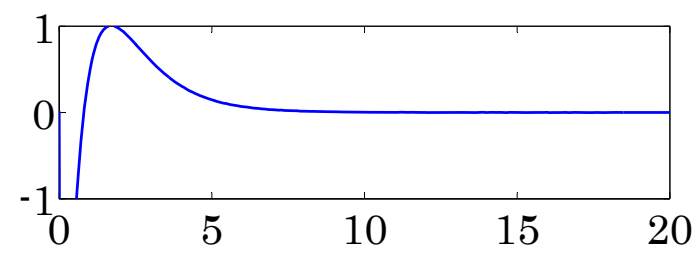

(b)

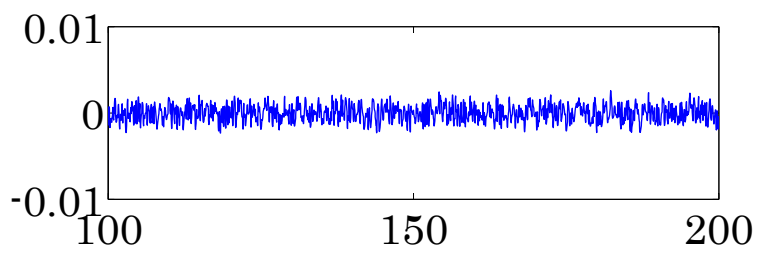

(d)

Time (sec)

Figure 11: Estimation errors of the updated-gain high-gain observer with significant identification errors where (a) the transient response of $v-\hat{v}$, (b) the transient response of $r-\hat{r}$, (c) the steadystate response of $v-\hat{v}$ and (d) the steady-state response of $r-\hat{r}$. The unit of $v-\hat{v}$ is $\mathrm{m} / \mathrm{s}$, and the one of $r-\hat{r}$ is $\mathrm{rad} / \mathrm{s}$.

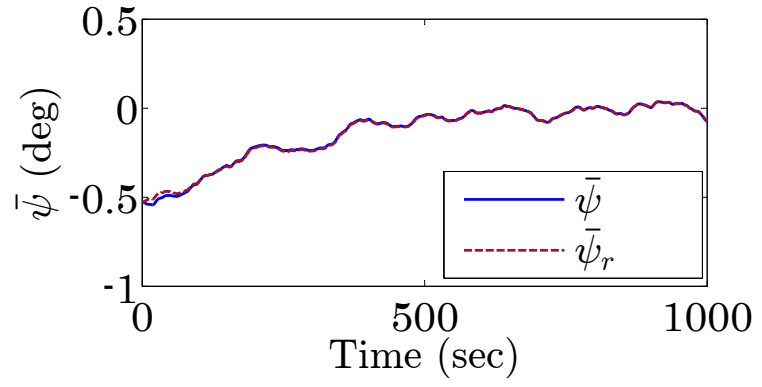

(a)

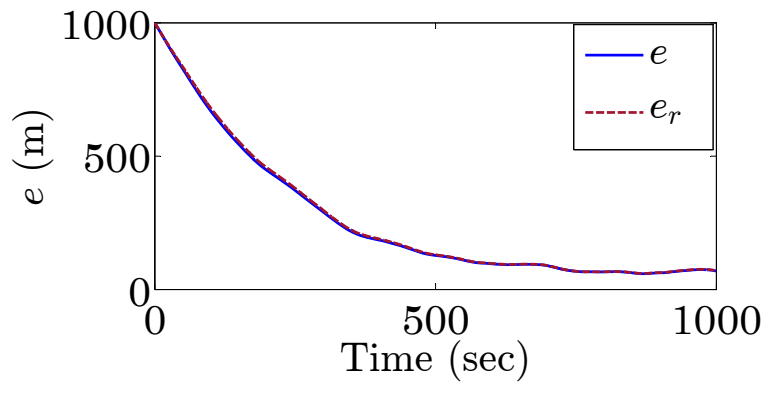

(b)

Figure 12: States with state feedback vs. output feedback with significant identification errors where (a) $\bar{\psi}$ and $\bar{\psi}$, (b) $e$ and $e_{r}$. 